\title{
Magnetic flux emergence into the solar photosphere and chromosphere
}

\author{
A. Tortosa-Andreu ${ }^{1}$ and F. Moreno-Insertis ${ }^{1,2}$ \\ ${ }^{1}$ Instituto de Astrofísica de Canarias (IAC), La Laguna (Tenerife), Spain \\ e-mail: abel.tortosa@gmail.com; fmi@iac.es \\ 2 Department of Astrophysics, Faculty of Physics, Universidad de La Laguna (Tenerife), Spain
}

Received 27 April 2009 / Accepted 24 June 2009

\section{ABSTRACT}

\begin{abstract}
Aims. We model the emergence of magnetized plasma across granular convection cells and the low atmosphere, including layers up to the mid-chromosphere.

Methods. Three-dimensional numerical experiments are carried out in which the equations of MHD and radiative transfer are solved self-consistently. We use the MURaM code, which assumes local thermodynamic equilibrium between plasma and radiation.

Results. In the photosphere, we find good agreement between our simulation predictions and observational results obtained with the Hinode satellite for the velocity and magnetic fields. We also confirm earlier simulation results by other authors. Our experiments reveal a natural mechanism of formation of twisted magnetic flux tubes that results from the retraction of photospheric horizontal fields at new intergranular lanes in decaying granules. In the chromosphere, we present evidence for the non-radiative heating of the emerging magnetized plasma due to the passage of shocks and/or ohmic dissipation. We study the formation of high-temperature points in the magnetic domain. We detect two types of points, classified according to whether they have a photospheric counterpart or otherwise. We also find evidence of those two types in Hinode observations.

Using Lagrangian tracing of a large statistical sample of fluid particles, we detect and study episodes of convective collapse of magnetic elements returning to the photosphere. On the other hand, we study the maximum heights reached by all tracers, magnetized or otherwise. Only a small fraction $(1.3 \%)$ of the magnetic elements reach the mid-chromosphere $(z>750 \mathrm{~km})$, while virtually no unmagnetized elements in the sample rise above the level of the reverse granulation (a few $100 \mathrm{~km}$ above the photosphere). We find that the rise into the chromosphere occurs in the form of successive jumps with intermediate stops rather than in a smooth continuous fashion and propose a tentative explanation of this behavior. Finally, also using Lagrange tracing, we document the creation of hightemperature points in the chromosphere via rising shock fronts.
\end{abstract}

Key words. Sun: magnetic fields - Sun: chromosphere - Sun: photosphere - magnetohydrodynamics (MHD) - radiative transfer Sun: granulation

\section{Introduction}

The emergence of magnetized plasma into the solar atmosphere after traversing the turbulent convection cells below the surface is one of the basic processes that determine the structure and dynamics of the observable domains of the Sun. Magnetic bipoles and, more generally, magnetic regions emerge from the solar interior on a wide range of length- and timescales, stretching from those of the largest activity complexes, with horizontal sizes comparable to the depth of the entire convection zone, through those of intermediate-sized active regions and ephemeral regions down to small-scale, granule-sized emergence events (as recently observed with Hinode/SOT by Centeno et al. 2007; Otsuji et al. 2007; Ishikawa et al. 2008; Orozco Suárez et al. 2008; Martinez Gonzalez \& Bellot Rubio 2009). Observationally, the use of Stokes polarimetry and the achievement of subarcsecond resolution in both ground-based and space observations have provided a wealth of knowledge concerning the emergence of magnetic field on different scales and at individual heights (additionally to the references just mentioned, see the papers by Martinez Pillet et al. 1994; Lites et al. 1995, 1998; De Pontieu 2002; Kubo et al. 2003; Harvey et al. 2007;
Lites 2009; Nagata et al. 2008; Okamoto et al. 2008; Shimizu et al. 2008; van Driel-Gesztelyi \& Culhane 2009). Yet, when rising, the magnetized plasma straddles the extremely inhomogeneous region constituted by the photosphere, chromosphere, and transition region, where density and pressure change by more than eight orders of magnitude in only a few $\mathrm{Mm}$ in height and temperature goes from photospheric values up to a million $\mathrm{K}$ in the corona. To observe a flux emergence event in its entirety one would need quasi-simultaneous measurements with detectors in the visible/IR, (E)UV, and X-ray ranges. This, and the stochastic nature of the flux emergence events, make it very difficult to put together a comprehensive observational picture of the flux emergence phenomenon.

The theoretical effort, on the other hand, has advanced using large $2 \mathrm{D}$ or $3 \mathrm{D}$ computer models of the emergence process that include the uppermost layers of the convection zone and a range of heights in the atmosphere. Two classes of models can be distinguished: a first branch includes the levels from a few $\mathrm{Mm}$ at the top of the convection zone all the way up to the corona. To ensure that the computing speed and data analysis are within reasonable bounds, these models strongly simplify the thermodynamics of the plasma by ignoring its interaction with the 
radiation field ; in most cases, heat conduction is also ignored (see as representative examples, e.g., Shibata et al. 1989; Matsumoto \& Shibata 1992; Nozawa et al. 1992; Matsumoto et al. 1993, 1998; Yokoyama \& Shibata 1995, 1996; Kusano et al. 1998; Fan 2001; Magara \& Longcope 2001, 2003; Manchester 2001; Abbett \& Fisher 2003; Archontis et al. 2004, 2005; Manchester et al. 2004; Miyagoshi \& Yokoyama 2004; Galsgaard et al. 2005, 2007; Murray et al. 2006, 2009; Arber et al. 2007; Moreno-Insertis et al. 2008). The neglect of the coupling with the radiation field in the energy equation limits the possibilities of detailed comparison with observational data in those regions where the radiative effects are important (most prominently the photosphere). On the positive side, these models lead to conclusions about phenomena such as the formation of current sheets at the interface between emerging and pre-existing flux systems in the atmosphere, field line reconnection, emission of high-speed, high-temperature jets in the corona, or plasmoid formation.

On the other hand, in the past few years a new generation of flux emergence models has appeared that solve the radiation transfer problem simultaneously with the MHD equations (Cheung et al. 2007a, 2008; Martínez-Sykora et al. 2008; see also Dorch et al. 2001). In the two papers by Cheung et al., the domain considered extends for $450 \mathrm{~km}$ and $300 \mathrm{~km}$, respectively, above the average $\tau_{5000}=1$ level, whereas, in depth, the box extends for $1.85 \mathrm{Mm}$ and $5.5 \mathrm{Mm}$, respectively. To start the emergence process, the authors planted a buoyant horizontal magnetic flux tube in the lower levels of the domain. Cheung et al. (2007a) considered cases with different field intensities ranging from 8500 to $2500 \mathrm{G}$, different levels of field line twist and magnetic flux between approximately $3 \times 10^{18}$ and $10^{19} \mathrm{Mx}$. When rising, the tubes of the weakest field intensity and lowest flux were strongly distorted by the convective flows; upon reaching the surface, a saltand-pepper pattern of vertical magnetic elements with mixed polarities resulted that had no simple bipolar region structure. On the other hand, the cases with higher initial field strength and higher total flux managed to distort the granulation pattern: a series of abnormally large and dark granules appeared when the magnetic domain reached the photosphere; these anomalous granules were arranged along a lane that reflects the geometry of the initial magnetic tube. In the later paper by Cheung et al. (2008), the tube had an initial total flux $1.6 \times 10^{20} \mathrm{Mx}$ with the buoyancy concentrated in the center of the box. Hence, the tube develops an $\Omega$-loop shape perturbed by the convective flows and yields a globally bipolar magnetic region at the surface, even though with a large amount of mixed polarity in its interior. A wealth of further features amenable to comparison with actual observations were obtained in these experiments, such as the convective collapse of photospheric flux tubes, cancellation of magnetic flux at the surface, and the existence of transient kilogauss horizontal fields. In both papers, the simplification of a gray atmosphere in local thermodynamic equilibrium (LTE) was used to model the radiation transfer.

In the more recent work by Martínez-Sykora et al. (2008), the experiments are carried out over a large domain in the vertical direction, including 1.4 Mm below and 14.6 Mm above the photosphere. They, therefore, include chromosphere, transition region and an extended domain in the corona. The equation of radiation transfer is solved using the opacity binning method (Nordlund 1982) and includes coherent scattering in the continuum following the method developed by Skartlien (2000). Additionally, optically thin radiative losses in the upper chromosphere and corona were included in the energy equation by means of source terms based on precalculated functions, in part using the non-LTE dynamical chromospheric models of Carlsson \& Stein $(1997,2002)$. The authors inject magnetic flux through the lower boundary either in the form of a magnetic tube (with different levels of field line twist in different experiments) or of a magnetized horizontal sheet. Of particular relevance to the current paper are the results concerning the chromosphere: cold volumes develop coinciding with (in fact, appearing slightly before) the arrival of the magnetized plasma there; the chromosphere and the transition region are pushed upward by as much as 3-4 Mm by the emerging plasma; bright points are formed in the periphery of the rising tube both at photospheric and chromospheric heights, with high values of magnetic field intensity, vertical velocity and vorticity.

The current paper builds upon the work of Cheung et al. (2007a), enlarging the physical domain of the experiment to include chromospheric layers (more precisely, the low and middle chromosphere). The focus of this paper is a detailed study of the phenomena taking place at chromospheric heights during the emergence process as well as a comparison of the evolution in the different layers from the photosphere to the midchromosphere. The paper starts with a brief description of the emergence of flux at the photosphere, especially concerning the magnetically modified granulation and the statistics of the horizontal and vertical field components (Sects. 3.2 and 3.3). Of special interest is the discovery of the formation of horizontal twisted flux tubes in the subphotospheric layers right below the downflow lanes that appear across decaying granules (Sect. 3.4). The twisted tubes are created as a result of the submergence of horizontal photospheric fields effected by the downflow: a $\mathrm{U}$-loop is formed in the subphotospheric levels, reconnection ensues and a twisted, roughly horizontal tube is formed.

The events at chromospheric heights are studied in detail in Sect. 4. The chromosphere prior to flux emergence in our experiment (Sect. 4.1) resembles in many respects the higher levels in the experiments of Wedemeyer et al. (2004), who studied the structure of the non-magnetic chromosphere with particular attention to its heating by means of rising shock fronts. In Sect. 4.2, three types of patterns developing in the flux emerging regions are described, namely: 1) extended cool patches, 2) hot filaments forming between adjacent regions, and 3) hightemperature points. Further subsections are devoted to the geometry and topology of the magnetic field from photosphere to chromosphere (Sect. 4.3), the heating of the magnetized plasma (Sect. 4.4), and the relation between chromospheric high- $T$ points, downflows, and magnetic concentrations observed by Hinode (Sect. 4.5): we identify, both in simulations and observations, two types of chromospheric high- $T$ points that differ in the nature of their photospheric counterparts.

A statistical study of the evolution of individual plasma elements involved in the emergence process is carried out in Sect. 5. To this end, Lagrange tracers initially located below the photosphere are used. Among other things, we study the average height reached by magnetized and unmagnetized elements in their rise (Sect. 5.2); we find that only a tiny minority of the initial tracers reach the chromosphere (less than $1 \%$ of the unmagnetized elements rise above $200 \mathrm{~km}$ in the atmosphere; only about $1 \%$ of the magnetized ones rise beyond $750 \mathrm{~km}$ in height). We also find that the magnetized elements reaching high levels in the chromosphere do not rise in a continuous fashion but, instead, go through a series of temporary stops along the rise. Finally, we show direct evidence for the origin of the high- $T$ points at chromospheric levels in terms of shock waves traveling upward in the middle of the magnetized cool patches resulting from the emergence (Sect. 5.3). 


\section{Methods, numerical setup, and initial and boundary conditions}

\subsection{Equations and numerical tools}

The numerical experiments in the present paper solve the equations of magnetohydrodynamics including radiation transfer. We use the customary MHD equations for the time evolution of the mass, momentum, and total energy densities and of the magnetic field (the induction equation). The equations include viscous and ohmic dissipation and a cooling term that corresponds to the exchange of energy between the plasma and the radiation field. The equations are solved using the MURaM code (Vögler et al. 2005), which includes 4th-order accurate spatial and time derivatives. The code also includes hyperdiffusivity algorithms to smooth discontinuous transitions, such as shocks. We use them for the viscous diffusion, while for the resistive terms the standard MHD expression is used. The radiation transfer problem is tackled by solving the radiation transfer equation,

$\frac{\partial I_{v}}{\partial s}=\kappa_{v}\left(S_{v}-I_{v}\right)$

along 24 rays passing through each grid point. In Eq. (1), $s$ is the arc-length along each given ray, $I_{v}$ and $S_{v}$ are the intensity field and the source function, and $\kappa_{v}$ is the opacity. In this paper, we use a single frequency point for the spectral dimension, and an LTE approach (Planck source function, Saha-Boltzmann equilibrium populations, no photon scattering). For the photosphere, the foregoing simplifications can provide useful results, since LTE is an acceptable approximation there and no detailed comparison with observational spectroscopic results is attempted. In the chromosphere, non-LTE effects may be of importance, e.g., in analyzing temperatures in the shocked plasma (see Sect. 6.2); we nevertheless also use LTE in those levels as a first step, to avoid that too many different physical effects operate simultaneously in the experiments, which would unduly complicate the analysis of the results. As an equation of state, we use the tables provided by the OPAL project (Rogers et al. 1996; Rogers \& Nayfonov 2002). Some elements undergoing quasi-adiabatic expansion in the chromosphere may reach the lower temperature limit of the OPAL table. For those elements, we set a minimum temperature around $2500 \mathrm{~K}$.

\subsection{Initial and boundary conditions}

We build the initial condition for the experiments in two steps, following the general scheme used by Cheung (2006). First, a non-magnetic model of stationary $3 \mathrm{D}$ convection is created that includes the topmost few megameters below the surface, the photosphere, and the low-mid chromospheric heights. We then introduce magnetic flux into the lower part of the box, which leads to the flux emergence episode.

In the present paper, for the non-magnetic convection model we use a plane-parallel stratification using the $1 \mathrm{D}$ vertical profiles of Spruit (1974) for the interior and VALC (Vernazza et al. $1981)$ for the atmosphere. The size of the box in the horizontal directions is $16 \mathrm{Mm} \times 12 \mathrm{Mm}(x, y:[-8,8] \times[-6,6])$. The vertical size is $3.8 \mathrm{Mm},(1.2 \mathrm{Mm}$ above, $2.6 \mathrm{Mm}$ below the solar surface). The surface, located at $z=0$, is determined as the level where the mean Rosseland optical depth is unity. The grid spacing is set at $20 \mathrm{~km}$ (height) and $50 \mathrm{~km}$ (horizontal), so that the numerical grid has $320 \times 240 \times 190$ points in $(x, y, z)$. To promote the convective instability, white noise at the $1 \%$ level is added to the specific internal energy in the vertical range $(-500,10) \mathrm{km}$.
Table 1. Set of parameters for the initial magnetic flux tube in the runs described in the paper.

\begin{tabular}{lcccc}
\hline \hline Label & $B_{0}(\mathrm{G})$ & $\beta_{0}$ & $\Phi_{0}\left(10^{19} \mathrm{Mx}\right)$ & Entropy prescription \\
\hline S1 & 7550 & 4 & 3.8 & Horizontal tube \\
S2 & 7550 & 4 & 3.8 & $\Omega$-loop \\
\hline
\end{tabular}

Note: $B_{0}$ and $\beta_{0}$ refer to the values at the tube axis, located $1.8 \mathrm{Mm}$ below the photosphere.

The system is allowed to evolve for 2 solar hours, yielding a thermally relaxed, statistically stationary state for the convection.

In the second step, a horizontal magnetic flux tube is added to the relaxed convection model. The tube axis is parallel to the $x$-axis and is located $1.8 \mathrm{Mm}$ below the surface at $y=0$. The field lines are twisted around the axis; using cylindrical coordinates $(r, \theta)$ centered on the tube axis, the longitudinal $\left(B_{1}\right)$ and azimuthal $\left(B_{\theta}\right)$ field components are given by

$\left\{\begin{array}{l}B_{1}(r)=B_{0} \exp \left(-\frac{r^{2}}{R_{0}^{2}}\right), \\ B_{\theta}(r)=\lambda \frac{r}{R_{0}} B_{1} .\end{array}\right.$

In Eq. (2), the parameter $R_{0}$ provides a measure of the tube radius and $\lambda$ for the field line twist. In this paper, we choose $R_{0}=400 \mathrm{~km}, \lambda=0.25$. The velocity distribution and the divergence of the stress tensor remain unchanged, so that, to compensate for the Lorentz force, the gas pressure distribution is adjusted in the magnetized domain. The actual values used for $B_{0}$ and the resulting plasma $\beta$ at the tube axis are given in Table 1.

The plasma in the tube is made buoyant by modifying its entropy and, consequently, its density. Two different cases are presented in this paper, called S1 and S2 in the following (see Table 1). For S1, the horizontal-tube experiment, buoyancy is imparted all along the length of the tube, while the perturbation decreases the further one goes away from the tube axis. To achieve that, the unperturbed entropy distribution, $s_{0}(x, r)$ is changed to: $s_{t}(x, r)=s_{0}(x, r)+\left[\bar{s}_{\text {upf }}-s_{0}(x, r)\right] \frac{B_{1}(r)}{B_{0}}$, where $\bar{s}_{\text {upf }}$ is the mean specific entropy of the upflows at the position of the tube axis. The buoyant tube is therefore expected to rise retaining a globally horizontal shape, even if more or less distorted by the convective flows. For S2, the $\Omega$-loop case, the buoyancy is concentrated in the central part of the tube and given by $s_{t}\left(x, r \leq R_{0}\right)=\bar{s}_{\mathrm{dwn}}+\left[\bar{s}_{\mathrm{upf}}-\bar{s}_{\mathrm{dwn}}\right] \cos ^{2}\left(\frac{x \pi}{L_{x}}\right)$, where $L_{x}=16 \mathrm{Mm}$ and $\bar{s}_{\mathrm{dwn}}$ is the mean entropy for the downflows near the visible surface $(z=-50 \mathrm{~km})$. The part of the tube close to $x=0$ is therefore underdense both with respect to the flanks and to the surrounding upflows and downflows. The tube then evolves into an $\Omega$-shaped structure, where the top of the $\Omega$ rises, its feet slowly descend toward the boundaries of the box.

As boundary conditions, we adopt periodicity on the sides, an open boundary at the bottom (balancing mass outflow and inflow, to guarantee mass conservation, as done by Vögler et al. 2005 or Cheung 2006), and a closed, stress-free boundary at the top. For the magnetic field, a zero vertical derivative condition for the horizontal components and constant vertical derivative for $B_{z}$ are imposed at the top and bottom boundary.

\section{Evolution in the interior and in the photosphere}

\subsection{Initial stages: evolution in the interior}

The initial properties of the magnetic tube, especially its initial field strength and twist, determine whether the rising magnetized plasma can resist the disrupting effect of the convective flows. 


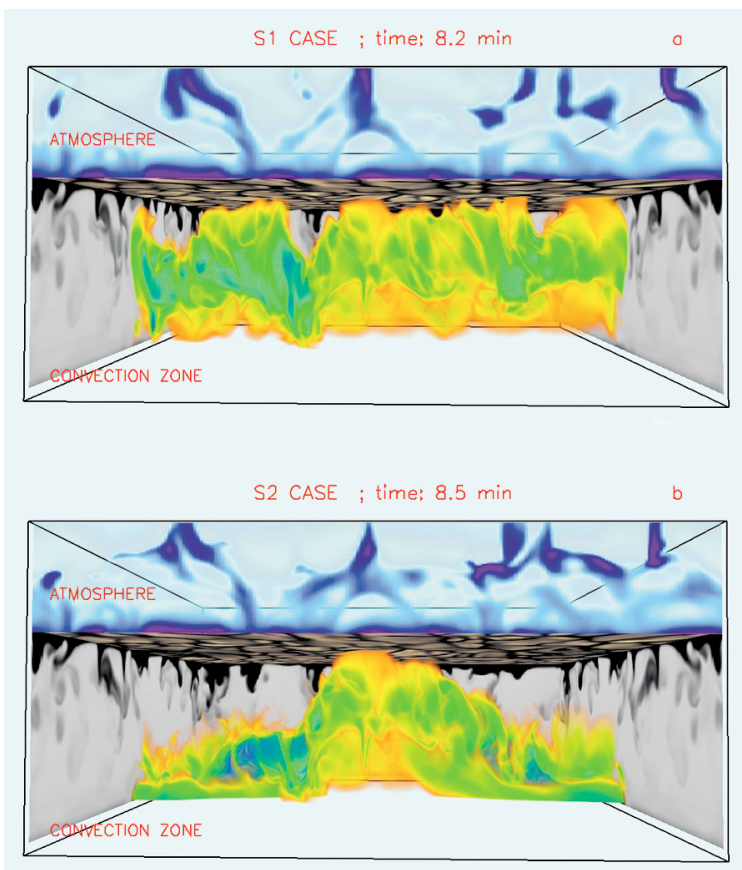

Fig. 1. Magnetized volume at the time of arrival of the magnetic flux at the surface (top: case S1; bottom: case S2). See explanations in the text (Sect. 3.1). Note that, for clarity, the vertical scale has been stretched.

Taking into account the local equipartition field strength with the convective flows (between 2 and $3 \mathrm{kG}$ at $z=-1.8 \mathrm{Mm}$ ) and on the basis of earlier estimates (Moreno-Insertis 1983; Fan et al. 2003; Cheung et al. 2007a), we expect our initial tube to be able to maintain its coherence along the rise, even if with a convectively perturbed shape. For both the S1 and S2 cases, the average upward velocity is $\sim 3 \mathrm{~km} \mathrm{~s}^{-1}$ and the tube needs $\sim 8$ min to reach the surface. For $\mathrm{S} 2$, the arrival at the surface is more gradual and takes a further $\sim 6$ min to complete. A visual impression of the subsurface evolution can be gained from Fig. 1, which in the lower two thirds of the box shows a volume rendering of $|B|$ at $t=8.5 \mathrm{~min}$, with colors from orange $(100 \mathrm{G})$ to solid blue $(5700 \mathrm{G})$. The horizontal map is drawn at the surface $(\langle\tau\rangle=1)$ and shows $v_{z}$ (dark: downflow; light: upflow). The volume rendering above the surface shows in blue the domains where $T>6500 \mathrm{~K}$. The vertical slices on the sides contain an entropy map (dark: low entropy; light: high entropy), clearly showing the penetration of the low-entropy downflows into the interior.

\subsection{Flux evolution in the photosphere: anomalous granulation}

Young unmagnetized granules in standard convection have a large pressure excess at their center that allows them to grow, pushing aside their neighbors (Stein \& Nordlund 1998). When the incipient granule carries a magnetic field of sufficient intensity, the additional push of the magnetic pressure can be expected to lead to anomalous granules, i.e., larger than usual cells. This is indeed the case in our experiments and those of Cheung et al. (2007a) (strong case) and Cheung et al. (2008).

In run $\mathrm{S} 1$ (the horizontal tube experiment), magnetized granules first appear at the photosphere as isolated cells around $t \sim 8$ min (upper-left panel of Fig. 2), growing to an abnormally large size (panels at 10.2 and 12.6 min in Fig. 2) with horizontal expansion velocity of $6-8 \mathrm{~km} \mathrm{~s}^{-1}$ (compared with $4-6 \mathrm{~km} \mathrm{~s}^{-1}$

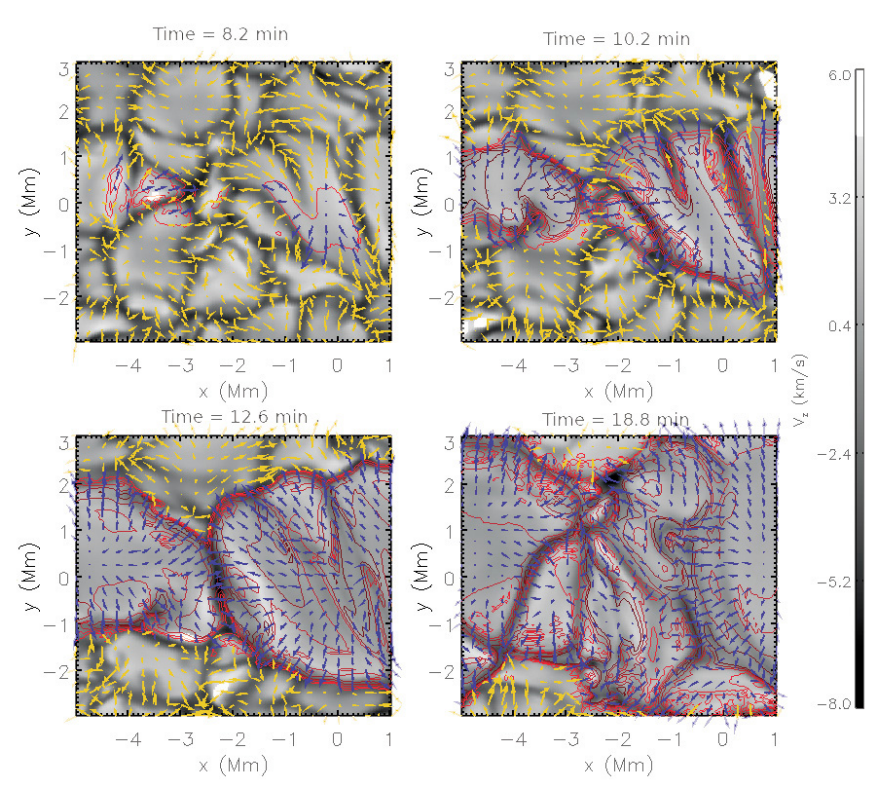

Fig. 2. Time evolution of the anomalous granulation for the S1 case. The gray shading is a Dopplergram (i.e., a color map for $v_{z}$ ) at the $\tau=1$ surface. The yellow and blue arrows represent the horizontal velocity field for the weakly-magnetic $(|B|<100 \mathrm{G})$ and magnetic granules $(|B| \geq 100 \mathrm{G})$, respectively. Contours for the magnetic field strength from $100 \mathrm{G}$ to $1000 \mathrm{G}$ are superimposed.

for young normal granules) and adopting an elongated shape. The anomalous granules reach a peak total pressure $20 \%$ larger than for normal granules, the difference being due basically to the magnetic pressure of the former.

At time $t \sim 12.5 \mathrm{~min}$, the collection of abnormal granules covers a lane parallel to the $x$-direction reflecting the initial tube orientation apparent in the upper panel of Fig. 1; in Fig. 2, one sees that the intervening unmagnetized granules have shrunk and disappeared (compare the two leftmost panels); each anomalous granule, in turn, is elongated in the $y$-direction. This anisotropy is due, first, to the competition between neighboring magnetized cells: they have a comparable pressure excess, so that the expansion in the direction away from the initial tube axis is favored. Additionally, when reaching the surface, the rising magnetic field points in the $y$-direction and the Alfven Mach number does not greatly exceed unity, so the Lorentz force tends to align the flows along the $y$-axis. The anisotropy is clearly noticeable in the horizontal velocities: a histogram of the azimuthal angle of $v_{\text {hor }}$ at this time $(t=12.5 \mathrm{~min})$ shows two marked peaks of some $7 \mathrm{~km} \mathrm{~s}^{-1}$ in the positive and negative $y$-direction. The average horizontal speed in the granule is $3.3 \mathrm{~km} \mathrm{~s}^{-1}$. Non-magnetized granules, on the other hand, have a fully isotropic distribution and the average speed is $v_{\text {hor }} \sim 2.6 \mathrm{~km} \mathrm{~s}^{-1}$.

The appearance of anomalous granulation in flux emergence regions can also be found in observational data. Anomalous, elongated granules can be directly seen in the movie by Hammerschlag et al. (2007), compiled with $G$-band spectral filter 430-431 nm observations taken with the Dutch Open Telescope (La Palma Spain). Our numerical results are also in agreement with the recent Hinode observations of a flux emergence episode by Otsuji et al. (2007). They show the presence of dark lanes of some $4^{\prime \prime}$ in length at the flux emergence site in the photosphere both in Stokes- $I$ and in the $G$-band. The values just reported for the horizontal speed in the magnetized domain (average: $3.3 \mathrm{~km} \mathrm{~s}^{-1}$; peak: $7 \mathrm{~km} \mathrm{~s}^{-1}$ ) are also compatible with the observed horizontal expansion of $3.8 \mathrm{~km} \mathrm{~s}^{-1}$. 

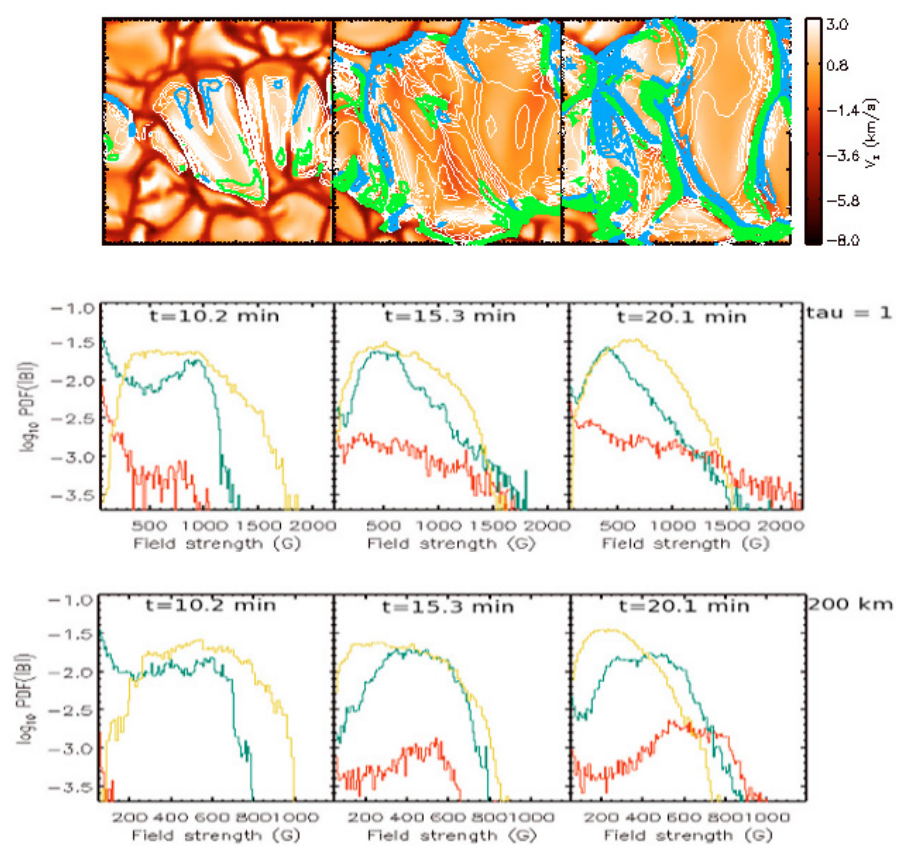

Fig. 3. Horizontal and vertical fields in the emerging flux region. The PDFs correspond to the horizontal field (green) and vertical field (red) at two heights: $\tau=1$ (middle row) and $z=200 \mathrm{~km}$ (bottom row). The yellow lines in them are the PDFs for the equipartition field, $\left(4 \pi \rho v^{2}\right)^{1 / 2}$. The top row contain color maps of the vertical velocity, with, superimposed, contours for the horizontal field strength $\left(B_{\text {hor }}\right.$, white) and for $B_{z}$ (blue: positive; green: negative).

The anomalous granules grow until about $t \sim 15 \mathrm{~min}$, reaching a maximum size of $\sim 7.5 \mathrm{Mm}^{2}$, several times the average for the non-magnetic granules. Thereafter, fragmentation sets in by means of a mechanism as for normal granules: the upflow velocity and energy transport to the surface decrease and some locations start to show an excess of radiative cooling. There, a downflow develops, and intergranular lanes form that cut across the anomalous granular cell.

Run S2 (the $\Omega$-loop case) also shows that the magnetic granules are abnormally large, although now, naturally, they form a cluster coinciding with the upper part of the $\Omega$-loop instead of a lane. The initial shape of the granules shows some elongation in the direction of the magnetic field, but this preference soon disappears; also, there is no privileged direction for the expanding horizontal velocities. The total magnetized area in the S2 experiment grows to occupy about $33 \mathrm{Mm}^{2}$ some $15 \mathrm{~min}$ after the first appearance of the magnetic field at the surface.

\subsection{Horizontal and vertical magnetic field in the photosphere}

In spite of the buffeting and distortion by the subsurface flows, we expect the magnetic field at the forefront of the rising magnetic domain to be oriented preferentially in the horizontal direction. This follows in part from the initial field distribution, but also from the predominance of the expansion in the horizontal directions along the rise. There is ample evidence in the literature for the relevance of horizontal fields in emerging flux regions, both from observations (Lites et al. 1996, 1998; Harvey et al. 2007; Lites 2009) and numerical experiments
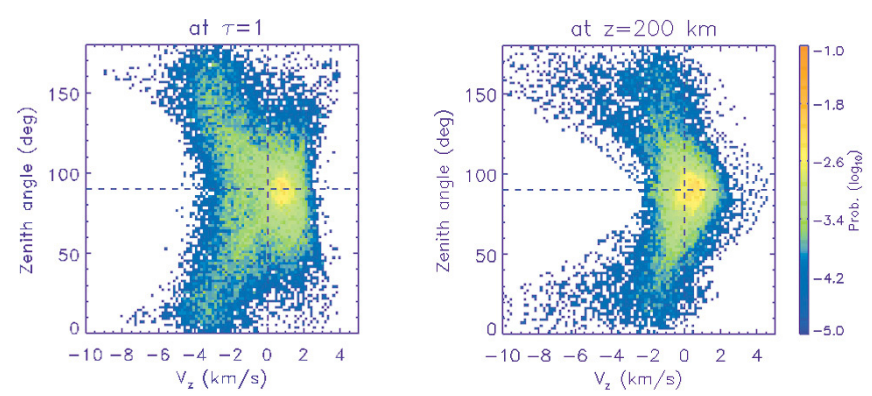

Fig. 4. JPDFs for the zenith angle and the vertical component of the velocity field at $t=20.2 \mathrm{~min}$. A zenith angle of $90^{\circ}$ corresponds to a horizontal magnetic field.

(Cheung et al. 2007a, 2008). Recently, using Hinode data, different groups have also detected horizontal fields in small-scale emergence episodes (Centeno et al. 2007; Otsuji et al. 2007; Ishikawa et al. 2008; Martinez Gonzalez \& Bellot Rubio 2009).

In our experiment, when the magnetized plasma first arrives at the surface yielding the large and elongated granules discussed in Sect. 3.2, we indeed find that the magnetic field is predominantly horizontal. The PDFs of Fig. 3, drawn for $\tau=1$ (middle row of panels) and $z=200 \mathrm{~km}$ (bottom row) illustrate the situation. The green and red curves in them show the number of pixels for a given $|B|$ where $B_{\text {hor }}>\left|B_{z}\right|$ (green) or viceversa (red). The green curves (horizontal fields) at time $10.2 \mathrm{~min}$ (leftmost panel in each series) have a maximum at $B=900 \mathrm{G}(\tau=1)$ and around $B=600 \mathrm{G}(z=200 \mathrm{~km})$, in either case with a far less numerous population of vertical fields. The top panel row in the figure contains color maps for the vertical velocity at the $\tau=1$ surface, with superimposed white contours for the horizontal magnetic field (contour range: $200<B_{\text {hor }}<1500 \mathrm{G}$ ) and color contours for $B_{z}$ (blue positive, green negative) in the range $300<\left|B_{z}\right|<2000 \mathrm{G}$. The first panel in that row $(t=10.2 \mathrm{~min})$ shows predominant horizontal fields in the cell interior with vertical footpoints in the intergranules. Five minutes later $(t \sim 15.3 \mathrm{~min}$, center $)$, the intergranular lanes are populated with vertical-field elements. Additionally, the anomalous granule is beginning to fragment and two vertically magnetized patches with positive and negative polarity, respectively, appear in its interior. Later still $(t \sim 20.1 \mathrm{~min})$, the color map shows an even more numerous population of vertically magnetized pixels in the intergranular lanes (including a new lane that has appeared in the process of fragmentation of the granule). We also note that the PDFs at either height show how the majority of pixels have horizontal fields at all times shown. Yet, at the most advanced time (rightmost panels), the vertical-field distribution reaches higher field strengths than the corresponding distribution for horizontal fields, namely (for $\tau=1$ ) $\sim 2.2 \mathrm{kG}$ versus $\sim 1.8 \mathrm{kG}$, respectively.

Using Hinode data, Ishikawa et al. (2008) have reported the observation of an emerging-flux region (EFR) in a remnant active region. The evolution that they describe bears a clear resemblance to the events explained above for our simulation, where emerging horizontal fields are flanked by vertical-field footpoints, even if their EFR occurs on a smaller spatial scale than in our case. The correlation between vertical velocity and field orientation is of particular interest. They mention downward vertical velocities up to $5 \mathrm{~km} \mathrm{~s}^{-1}$ coinciding with the vertical-field structures and upward flows of some $2 \mathrm{~km} \mathrm{~s}^{-1}$ in the horizontal fields. This matches well the joint probability density function (JPDF) between vertical velocity and magnetic field orientation obtained in our experiment, shown in Fig. 4. It may also be of 


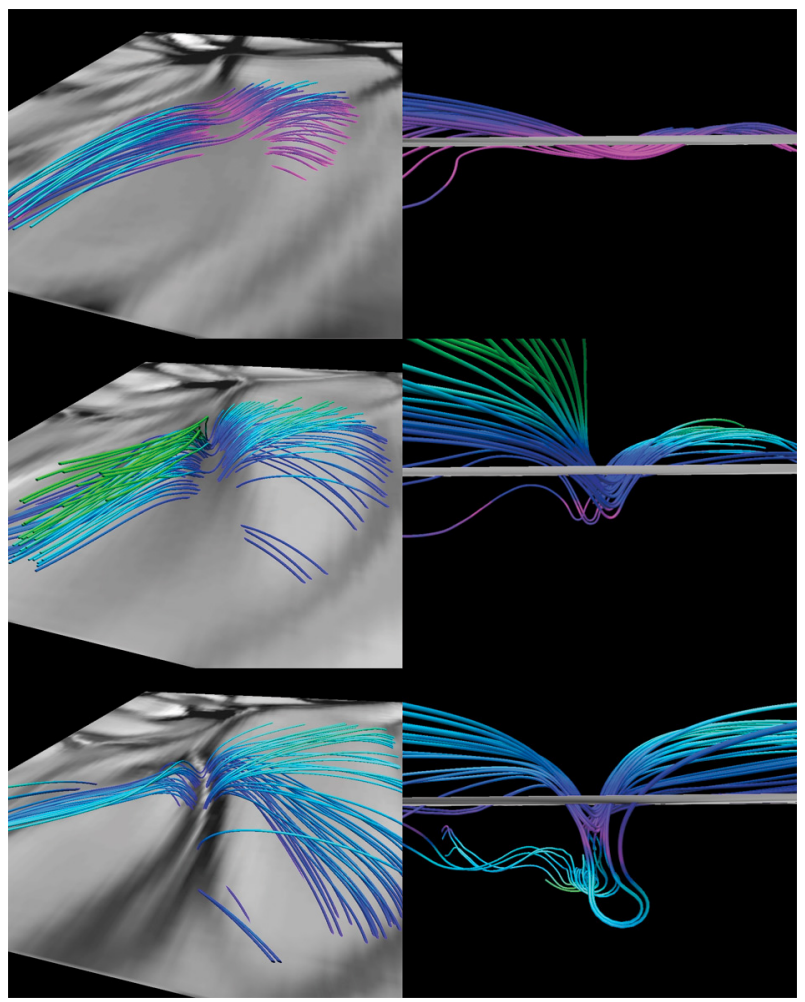

Fig. 5. Formation of U-loops below intergranular lanes: the downflow pulls down the initially horizontal field line and a corridor of U-loops is formed. Left: view from above; right: side view. Time advances from top to bottom in the figure.

interest to compare the JPDF for $z=200 \mathrm{~km}$ (right panel) with the JPDF at $\tau=0.1$ in Fig. 9 of Cheung et al. (2007a), calculated for their weak-field case: our JPDF has more clearly defined features, such as a more concentrated probability maximum and well developed vertical-downflow wings, this possibly being a consequence of the higher initial field strength of the magnetic tube in our experiment.

\subsection{Submergence of horizontal fields: formation of U-loops and twisted flux tubes}

The fragmentation of the granulation pattern discussed in Sect. 3.2 yields new intergranular lanes across the decaying cell, with associated downflows. The horizontal field that stretched across the old granular cell is thereby pulled down by the downflows toward the subsurface layers so that the field lines adopt a $U$-loop shape. This can be seen in Fig. 5, which shows the evolution of a bunch of field lines from the initiation of the process (topmost panels) to a time when the U-loops are already formed (bottom panels); both a view from above (left column) and from the side (right column) are provided. The left panels show the formation of a new intergranular lane, visible as an increasingly dark corridor in the velocity gray-scale map, in a region where the field was predominantly horizontal above the surface. On the right, the effects of the downflows on the field lines below the surface becomes apparent: the field lines are dragged down by the flows and two neighboring nearly vertical stretches of opposite polarity are formed not far below the surface, a nearly closed U-loop being evident in deeper layers.

The penetration of the mass downflows and field lines into the interior layers leads to the formation of overdense regions with a roughly horizontal, tube-like shape parallel to the
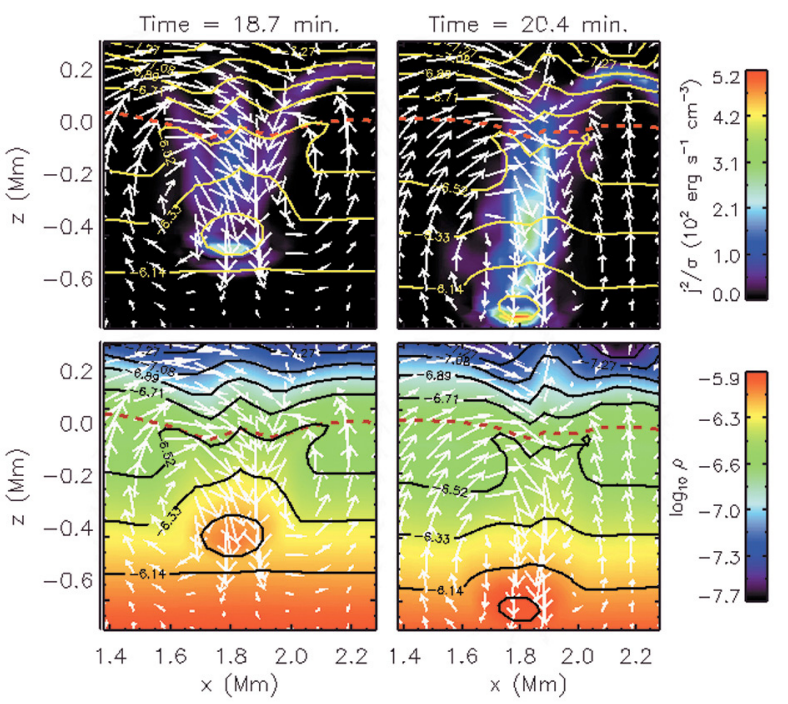

Fig. 6. Time sequence of a vertical cross-section of the box, along the direction of the tube axis, at $y=0.6 \mathrm{Mm}$. The upper and lower panels show the color maps of $j^{2} / \sigma$ and $\log _{10} \rho$, respectively. The arrows represent the projected velocity field. The solid isolines correspond to the density and indicate the formation and evolution of the ball-like overdense patch. The red dashed lines show the line of $\tau=1$.

intergranular lane. Since field lines of opposite polarity are brought close to each other (as is apparent in the bottom-right panel of Fig. 5), high levels of current density are produced and reconnection follows. Both phenomena can be seen in Fig. 6, which shows a time series for $t=18.7$ and $20.4 \mathrm{~min}$. The bottom panels show color maps of $\log \rho$ on a vertical plane that cuts across the intergranular lane. The excess density expected in any granular downflow adopts the shape of a ball-like region in this 2D plot (a tube-like region, if viewed in 3D). The overdense region (e.g., at $[1.8,-0.4] \mathrm{Mm}$ in the bottom-left panel) has a factor of 2 excess density compared with the surroundings at the same horizontal level. The top panels contain color maps of $j^{2} / \sigma$, where $\sigma$ is the coefficient of ohmic resistivity. In the figure, the formation of a current sheet coinciding with the downflow lane is apparent and is coherent with the opposite polarities being brought close to each other in the downflow region.

Magnetic reconnection between the vertical legs of the U-loop is the natural follow-up to this process. Field lines of opposite polarity are brought close to each other, reconnect, and two disjoint field regions result, one close to the surface, the other containing the bottom part of the former U-loops. This is fully three-dimensional reconnection. For instance, the bottom of the U-loops yield a quasi-horizontal twisted flux tube below the surface that runs almost parallel to the intergranular lane at the photosphere. This is illustrated in Fig. 7, which contains a view seen from below the surface of the evolution of the field lines that are being pushed down by the downflow. In the figure (from top to bottom), a twisted magnetic flux tube is clearly seen to form roughly below the intergranular lane, several $100 \mathrm{~km}$ below the surface, with a transverse size similar to the width of the intergranule. Simultaneously, the pinching off of the vertical part of the U-loops yields reconnection outflows in the vertical direction superposed on the general downflow. The plasma $\beta$ in those levels is not too large, so that these outflows are clearly detectable in the simulation. The upgoing outflow yields a very narrow lane of $v_{z}>0$ (i.e., white in Fig. 5) at the center of the intergranular corridor at the surface: this is apparent in the bottomleft panel of Fig. 5 and can be confirmed in Fig. 6 by means of 

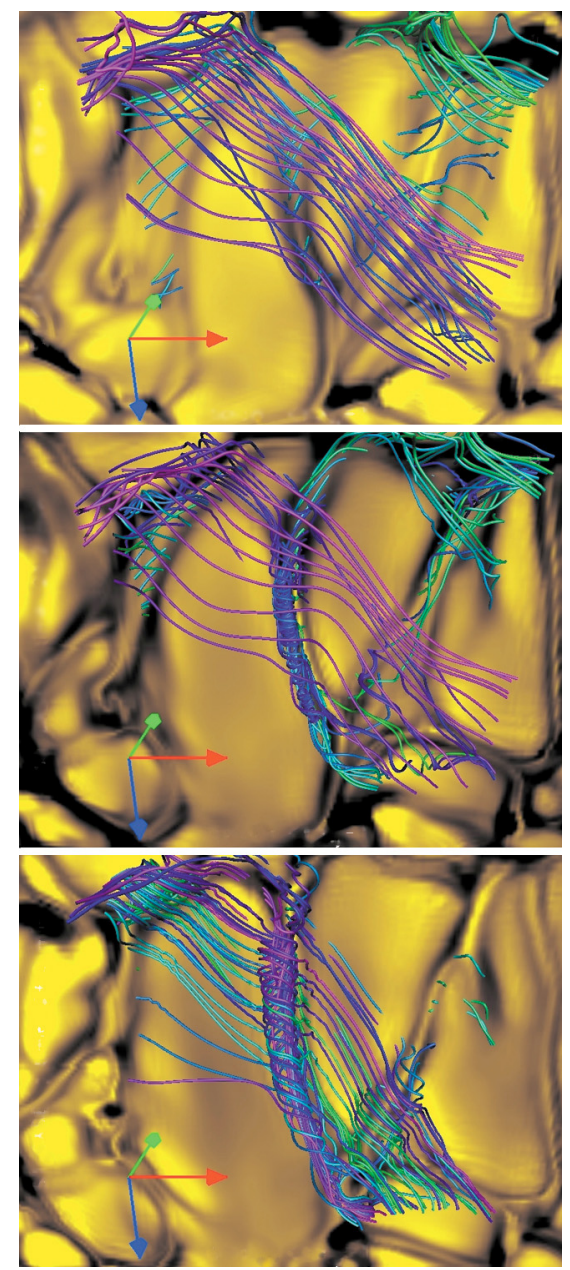

Fig. 7. Subsurface formation of a small magnetic twisted flux tube. The images show the time evolution of the magnetic field lines below the visible surface as seen when looking up from below the photosphere. The color maps correspond to the vertical component of the velocity field, from -3 (black) to 2 (yellow) $\mathrm{km} \mathrm{s}^{-1}$, at $\langle\tau\rangle_{x y}=1$ and illustrate the granulation pattern. The colors of the lines identify different seeds. The times are $18.8 \mathrm{~min}$ (top), $21.1 \mathrm{~min}$ (middle) and $24.0 \mathrm{~min}$ (bottom).

the white arrows close to the surface at $t=20.4 \mathrm{~min}$ (panels on the right).

\section{The chromospheric layers}

\subsection{The chromosphere before the emergence}

The chromosphere is a highly dynamical region, so it is not simple to set precise limits to it in terms of fixed geometrical heights or a fixed temperature stratification (Carlsson 2006; Judge 2006; Rutten 2007). In our experiment, we call the chromosphere (or, rather, the low-mid chromosphere) the region from $z=500 \mathrm{~km}$ to the top of the box $(z=1200 \mathrm{~km})$. Within it, we differentiate between the low chromosphere $(500 \mathrm{~km}<750 \mathrm{~km})$ and the mid-chromosphere for layers at higher altitudes (see Fig. 8).

Our initial 3D model of the chromosphere, i.e., the result of reaching stationary convection in lower layers before introducing the magnetic flux tube, is strongly reminiscent of the results of Wedemeyer et al. (2004): extended volumes of cool plasma coexist with a filamentary hot gas network, in a very

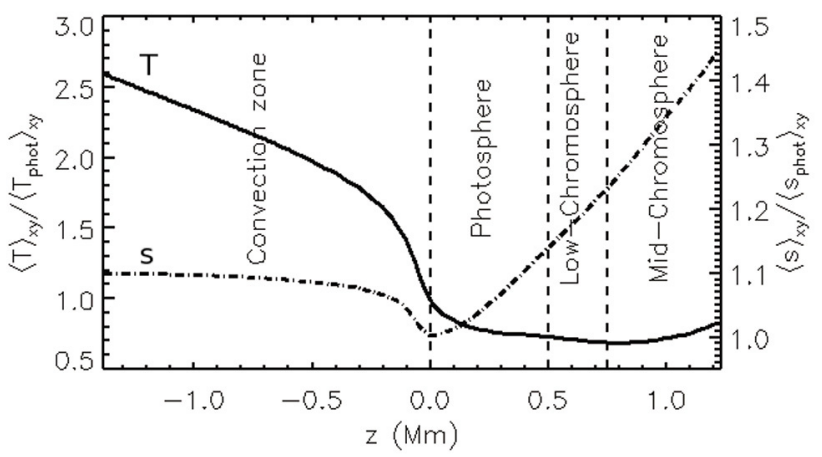

Fig. 8. Horizontal averages of temperature (solid) and entropy (dotdashed) from the convection zone to the mid-chromosphere for the stationary-convection stage before the emergence. Both quantities are expressed in units of the horizontal average at $\tau=1, T_{\text {phot }}$ and $s_{\text {phot }}$.

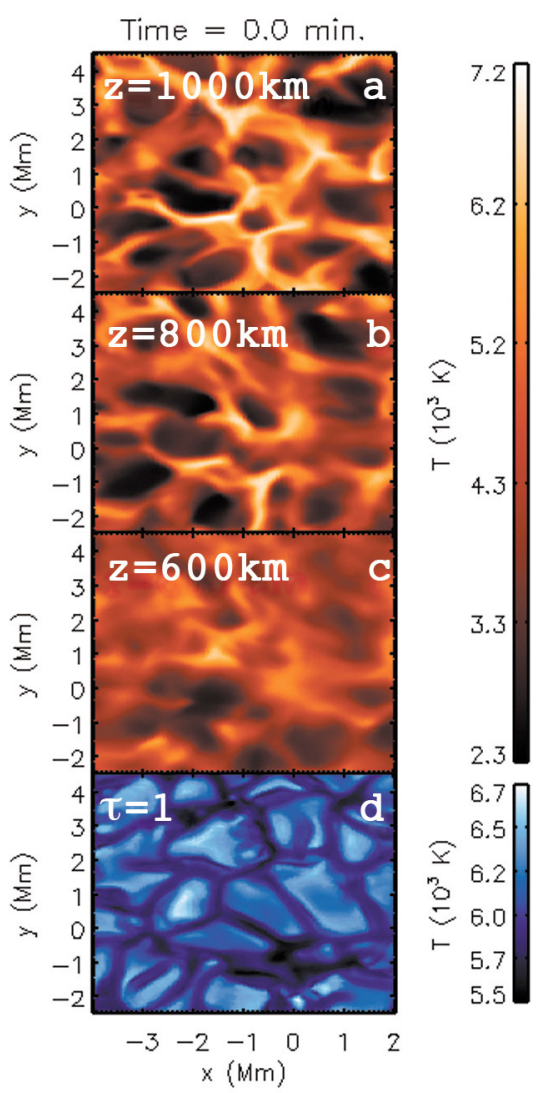

Fig. 9. Chromospheric thermal structure before the emergence. The colors maps from a to c correspond to 2D slices of temperature at the chromospheric heights $z=1000,800$ and $600 \mathrm{~km}$, respectively. The bottom panel is shown for comparison and illustrates the temperature of the granulation pattern at the visible surface.

dynamic and intermittent pattern (see top panel, Fig. 9). This thermal structure is completely determined by shocks resulting from the excitation of acoustic waves by convective motions in the lower layers. The shocks compress and heat the gas in the filaments (hot component, between $\sim 6000$ and $7000 \mathrm{~K}$ ), whereas the quasi-adiabatic expansion of the plasma in the post-shock region results in a decrease of the temperature (cool component, $\sim 3000 \mathrm{~K}$ ). The cool volumes have the size of normal granules (e.g., at $[x, y]=[-2,0.5]$, top panel, Fig. 9). Some of the hot 

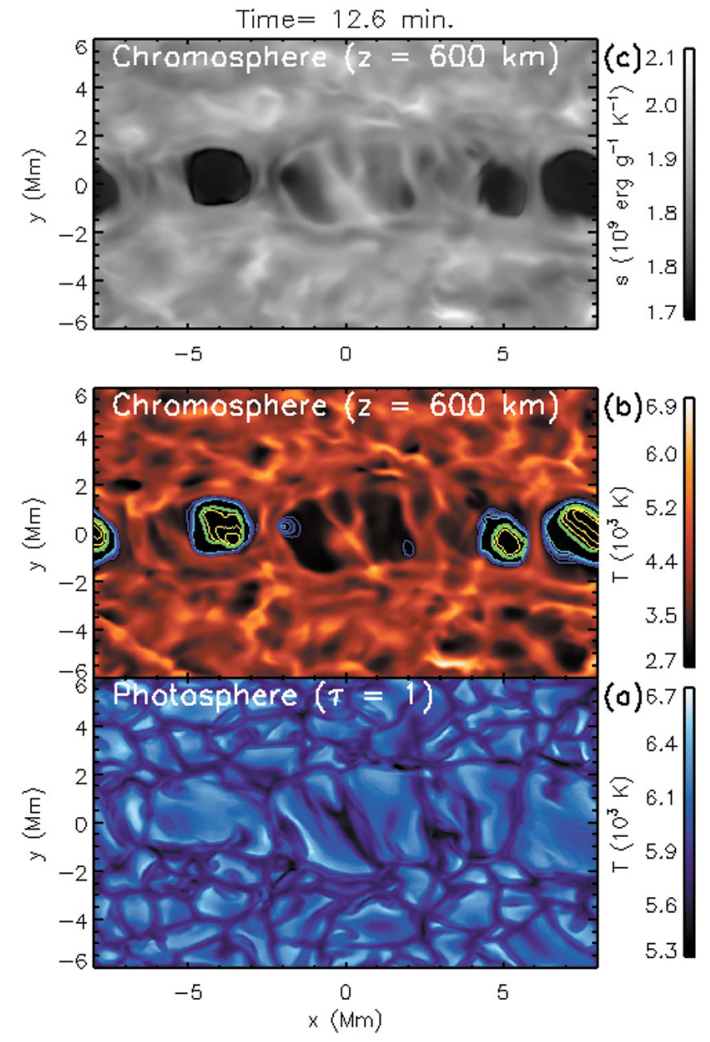

(a) 6.7

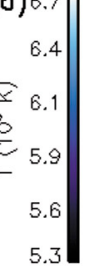

Fig. 10. Thermal structure when the magnetic field reaches the low chromosphere. The central and bottom panel contain temperature maps at $z=600 \mathrm{~km}$ and $\tau=1$, respectively. The top panel shows the entropy distribution at $z=600 \mathrm{~km}$. The middle panel contains isolines of plasma $\beta$ (green: $\beta=1$; blue: $1<\beta \leq 100$; yellow: $0.6 \leq \beta<1$ ).

filaments are thin and sharply defined (e.g., at $[x, y]=[-2,-0.3]$ in the same panel); others are cooler and fuzzier (e.g., at $[x, y]=$ $[0.3,2])$. The patterns change on a shorter timescale in the chromosphere than in the photosphere. As a consequence, it is difficult to establish a direct correlation between the chromospheric structures and the granulation, as illustrated by comparing panels (a) and (d) of Fig. 9.

The thermal bifurcation just described becomes more pronounced the higher we rise in the chromosphere. In the lowermost few hundred kilometers, the shocks remain weak, and the thermal components are not clearly distinguishable (Fig. 9, panel (c)). Higher up, the shocks become stronger and small hot filaments appear (panel (b)). At $z=1000 \mathrm{~km}$ (panel (a)), the hot and cool components can be clearly identified (with extreme values of 2400 and $7100 \mathrm{~K}$ ). In agreement with Wedemeyer et al. (2004), who used an LTE approach, and the detailed 1D nonLTE radiative models by Carlsson \& Stein (1995), the resulting horizontal average temperature (solid line in Fig. 8) shows neither the significant increase with height nor the pronounced minimum obtained in the semi-empirical models of Vernazza et al. (1981). The qualitative agreement with the non-LTE models, in particular, suggests that our experiments capture some of the fundamental properties of the solar chromosphere, in spite of the simplified treatment of the opacities and radiative transfer.

\subsection{Patterns resulting from the emergence}

\subsubsection{Arrival of magnetic flux at the chromosphere}

In the $\mathrm{S} 1$ case, the arrival of magnetized plasma at the lowest chromospheric levels ( $z$ between 500 and $600 \mathrm{~km}$ ) occurs at
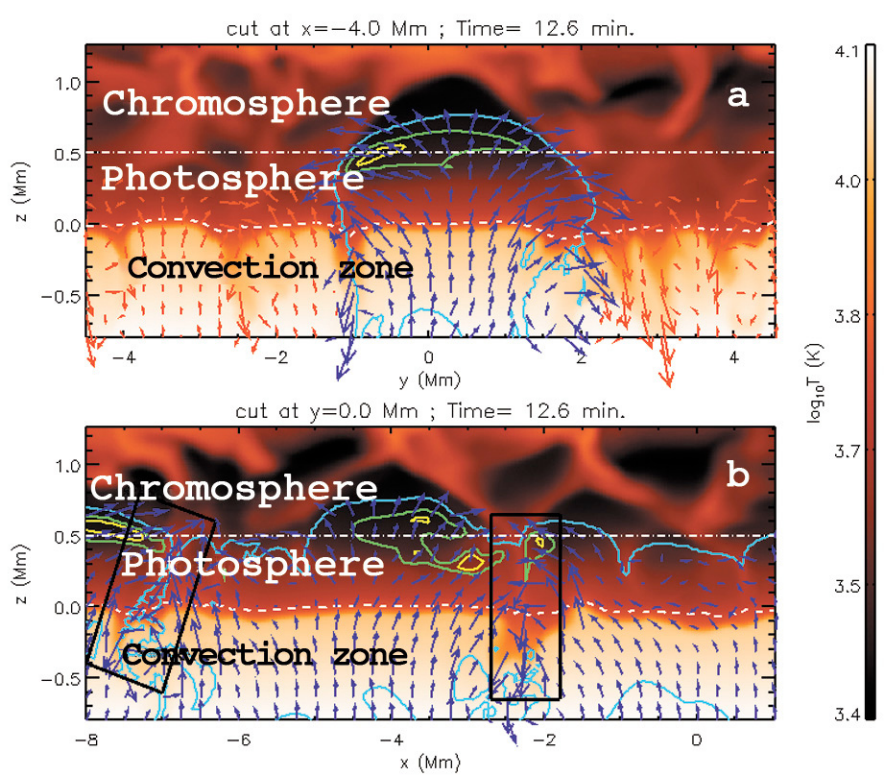

Fig. 11. Fountain-like flows within the magnetized plasma at chromospheric heights. The panels show color maps of $\log _{10} T$ on vertical cuts along the $x$ and $y$ directions that intersect the cool patch at $[x, y]=[-4,0] \mathrm{Mm}$ in Fig. 10. The solid isolines correspond to the plasma $\beta$ (same color code as in Fig. 10). The white dashed line near $z=0 \mathrm{~km}$ indicates $\tau=1$. Other symbols: see text.

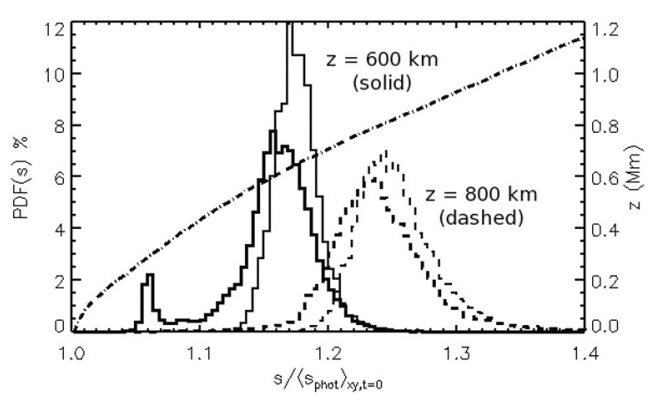

Fig. 12. PDF of the specific entropy at $z=600 \mathrm{~km}$ (two leftmost distributions) and $z=800 \mathrm{~km}$ (rightmost distributions). Thin lines: $t=0$; thick lines: $t=12.6$ (same times as for Figs. 11 and 10). The dotdashed line is the horizontal average of the specific entropy at $t=0 \mathrm{~min}$, $\langle s\rangle_{x y}(z, t=0)$. The entropy values are expressed in units of the horizontal average at $\tau=1$ for $t=0 \mathrm{~min},\left\langle s_{\text {phot }}\right\rangle_{x y, t=0}$.

$t \sim 12.6 \mathrm{~min}$, i.e., some $5 \mathrm{~min}$ after the tube has reached the visible surface. The patterns that develop in the chromosphere (Fig. 10) clearly reflect the distribution, morphology, and flows of the magnetized regions in the photosphere. In the temperature and entropy maps at $z=600 \mathrm{~km}$ (middle and upper panels), individual cool patches with $T \sim 3000 \mathrm{~K}$ appear superimposed on the pre-existing chromospheric pattern described in Sect. 4.1. Each cool patch is associated with an anomalous granule at the visible surface, as is apparent when comparing the central and bottom panels of the figure.

Figure 11 shows color maps of the logarithmic temperature on vertical cuts along the $y$-axis (panel (a)) and along the $x$-axis (panel (b)) that intersect the cool patch at $[x, y]=[-4,0] \mathrm{Mm}$ in the central and top panels of Fig. 10. The arrows correspond to the velocity field for the magnetized plasma (blue) and the non-magnetized granules (red). The top panel of Fig. 11 shows that the fountain-like flows of the emerging magnetized plasma 

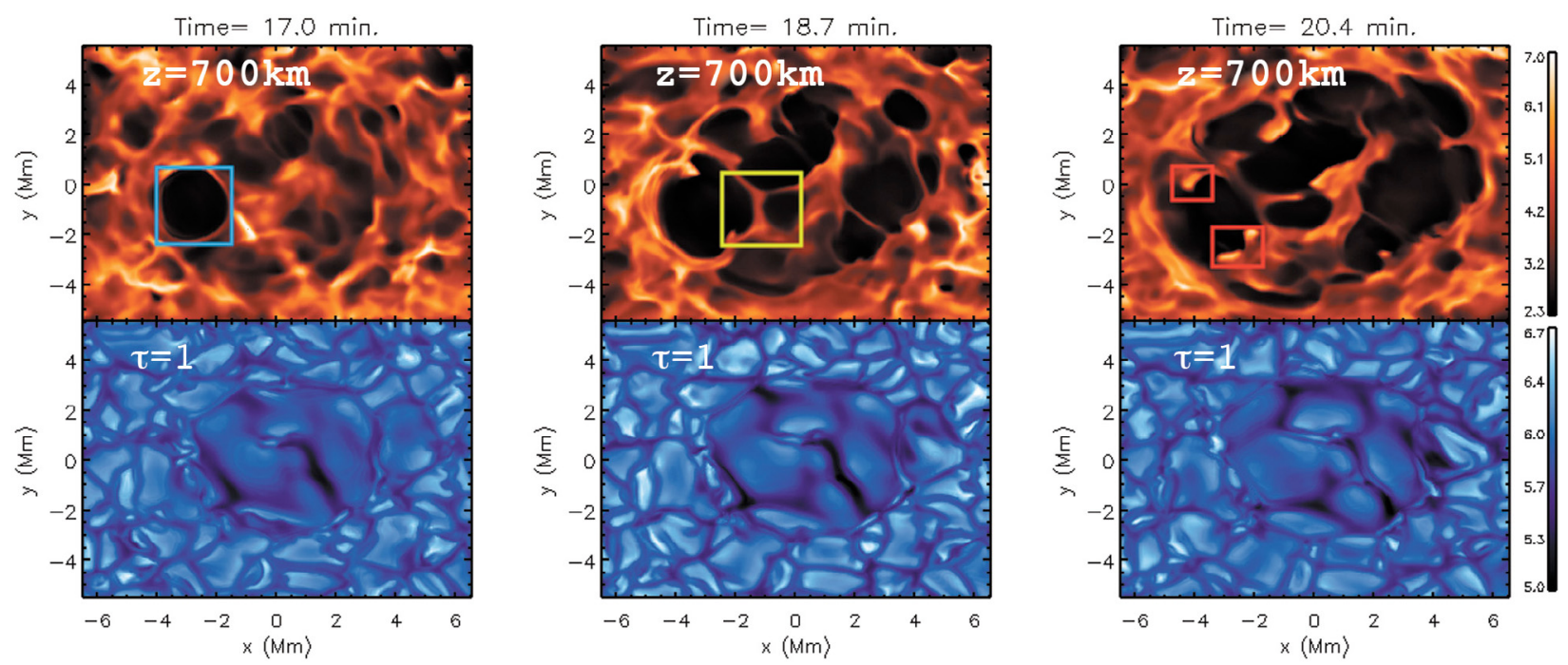

Fig. 13. Time evolution of the chromospheric temperature pattern during the emergence of the magnetic field for the $\mathrm{S} 2$ run. The color maps are drawn for the temperature (in $10^{3} \mathrm{~K}$ ) at the visible surface (lower panels) and at $700 \mathrm{~km}$ in the chromosphere (upper panels). The different types of thermal structures described in the text are highlighted with color boxes.

(blue arrows) reach well above the reversal level of normal granules. Typical rise speeds at the center of the cool patch are $1 \mathrm{~km} \mathrm{~s}^{-1}$. The sideways expansion reaches maximum speeds of about $10 \mathrm{~km} \mathrm{~s}^{-1}$. At the edges of the magnetized plasma (e.g., at $y=-1 \mathrm{Mm}$ ), concentrated downdrafts are formed that extend all the way down to the surface, connecting to the intergranular regions there. Other examples of chromospheric downdrafts are outlined by the black squares in the lower panel of the figure. On the other hand, Fig. 11 shows how the rising material pushes weakly magnetized plasma ahead of itself. This can be seen by means of the plasma $\beta$ isolines in the figure (e.g., those in the cool volume in the top panel). This also explains the widely varying values of $\beta$ in the cool patches of Fig. 10: those toward the center of the figure have high- $\beta$ values and correspond to the weakly magnetized upper part of a rising magnetic region with $T<3000 \mathrm{~K}$, whose top is reaching the $z \sim 1000 \mathrm{~km}$ level.

The initial evolution of the field in the chromosphere is basically adiabatic: the expansion of the plasma along the rise causes a drastic decrease in its density and temperature, which goes below the radiative equilibrium level. The opacities then become low enough for the radiative times to be much longer than the typical dynamical timescales. As a result, the entropy in the cool patches of Fig. 10 (see top panel) is significantly lower than the average value at that height. This can be determined quantitatively by means of Fig. 12, which contains PDFs for the specific entropy at two heights in the chromosphere $(z=600 \mathrm{~km}$, solid, and $z=800 \mathrm{~km}$, dashed), before $(t=0$, thin line $)$ and during $(t=12.6 \mathrm{~min}$, thick line $)$ the arrival of the magnetic field. The PDFs for the higher level have a higher-entropy peak and a larger spread. This agrees with the average entropy of the unperturbed atmosphere (dashdotted line) and with the larger heating ability of the shocks the higher they rise in the chromosphere. The profile for the later time at $z=600 \mathrm{~km}$ has a conspicuous low-entropy secondary peak that corresponds to the recently arrived magnetized material. Checking with the dot-dashed curve, we see that this peak corresponds to the average entropy at around $z \sim 275 \mathrm{~km}$, providing a general indication that the magnetized material has evolved isentropically from roughly that height. On the other hand, the majority of the non-magnetized plasma does not rise above the level of the reversed granulation (as demonstrated later, in Sect. 5.2.1 and Fig. 21), which explains the absence of low-entropy bins at the stages prior to emergence.

\subsubsection{Cool patches, hot filaments, and high-temperature points}

The evolution of the magnetized plasma in the chromosphere produces a variety of thermal structures: 1) irregular and extended cool patches, 2) hot filaments localized between two emerging regions, and 3) high-temperature points. Figure 13 shows the time evolution of the temperature pattern at the visible surface and at $700 \mathrm{~km}$ in the chromosphere for the S2 run. The most apparent structures are the cool patches (such as the one framed in blue), whose early stages were discussed in Sect. 4.2.1. Their characteristic sideways expansion velocity is $8-10 \mathrm{~km} \mathrm{~s}^{-1}$ and the upflow speed is $1-2 \mathrm{~km} \mathrm{~s}^{-1}$. They are always correlated with the anomalous granulation pattern on the surface (Fig. 13, lower panels).

The second type of thermal structure is a fragmented network of relatively hot and narrow filaments outlining the individual magnetic patches inside the magnetic volume, such as those highlighted by the yellow square in Fig. 13. They are created by the compression following the collision of neighboring cool magnetic patches as they expand along the rise. Hence, their origin and properties are different to those of the hot component of the initial, non-magnetic chromosphere. These filaments are also the site of comparatively large magnetic gradients, which have associated currents and Joule dissipation that cause an additional temperature increase as time proceeds. Their temperature is in an intermediate range $(\sim 4000 \mathrm{~K}$ to $\sim 6000 \mathrm{~K})$. Their plasma $\beta$ is significantly above unity when they are formed, while $\beta \sim 1$ toward the end of the simulation.

The last type of thermal structure is a collection of hightemperature points at the edges of some of the cool patches (see, e.g., the examples within the red squares on the right in Fig. 13). As for the filaments, their nature and properties differ from the hot component of the original chromosphere. A more detailed description of these high-temperature points will be given in Sect. 4.5.

We also find the same types of chromospheric patterns (cool patches, hot filaments, high- $T$ points) for the S1 run, with the 


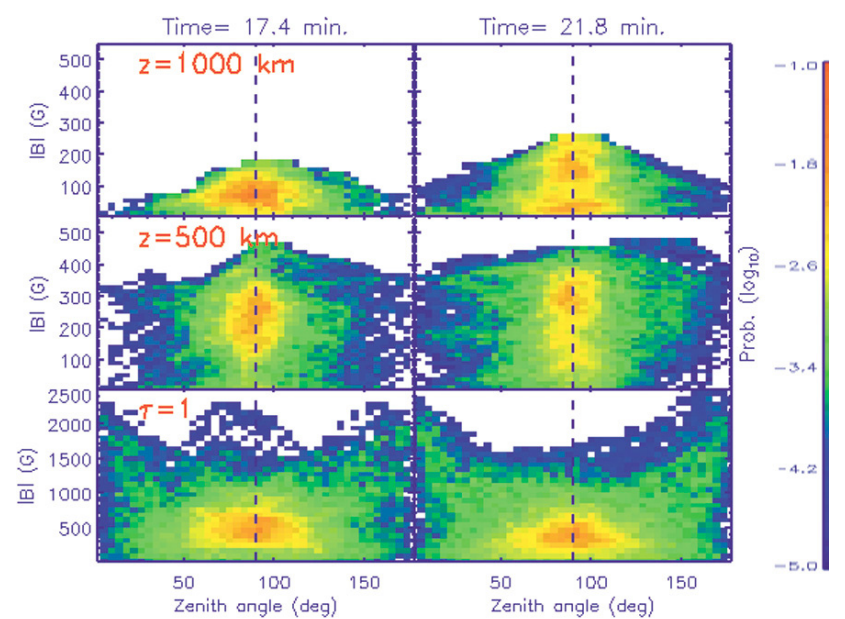

Fig. 14. JPDF for the magnetic field strength and zenith angle for various heights in the low atmosphere for the $\mathrm{S} 1$ run. The colors correspond to the probability density. The angles are measured from the vertical direction: the dashed vertical line at $90^{\circ}$ corresponds to the horizontal direction. The sample used contains those pixels with $B>50 \mathrm{G}(\tau=1$ panel) and $B>10 \mathrm{G}$ (chromospheric heights).

exception of the lane-like arrangement of the cool patches. The high- $T$ points here are found at the edges of the lane. Values of $T$ and plasma $\beta$ are similar to those in the previous paragraphs.

\subsection{Magnetic field intensity and orientation}

As in the photosphere, the emerging magnetized volume reaches the chromosphere with comparatively strong horizontal fields. This is reflected in the JPDFs between field strength and zenith angle shown in Fig. 14. In the topmost row, we see that, by the time the emerging field reaches the $z=1000 \mathrm{~km}$ level ( $t \sim 17.4 \mathrm{~min}$ ), most fluid elements there have $B \sim 100 \mathrm{G}$ and horizontal orientation (zenith angle around $90^{\circ}$ ). As time proceeds (right column), the slots of the JPDF corresponding to inclined or vertical fields become increasingly occupied.

A visual impression of the field line geometry at this stage can be gained from Fig. 15, which shows a collection of field lines calculated from a random distribution of points in a flux concentration at $z=400 \mathrm{~km}$. Horizontal planes at $\langle\tau\rangle_{x y}=1$ (yellow) and $z=500 \mathrm{~km}$ (blue) are included, both containing a color map of $v_{z}$. The panels show how the field lines are almost vertical between the top plane and the subphotospheric levels, coinciding at all those heights with a concentrated downflow. At heights above $z \sim 500 \mathrm{~km}$, the field lines become horizontal and the flux concentration is lost. This agrees with the JPDFs of Fig. 14, in which there is a transition between a twohorned distribution at the lower levels (with strong, kilogauss vertical fields) and a mountain-top kind of distribution for the highest levels, with only weak highly inclined fields. The panels of Fig. 15 also reflect the transition from a nearly statistically stationary situation at the surface to a distribution that evolves in time as the rising flux reaches the highest levels in the box.

\subsection{Chromospheric heating of the magnetized plasma}

In Sect. 4.2.1, we have seen that the magnetized plasma reaches the chromosphere with low entropy and temperature (see the low-entropy secondary maximum in Fig. 12). As time proceeds,

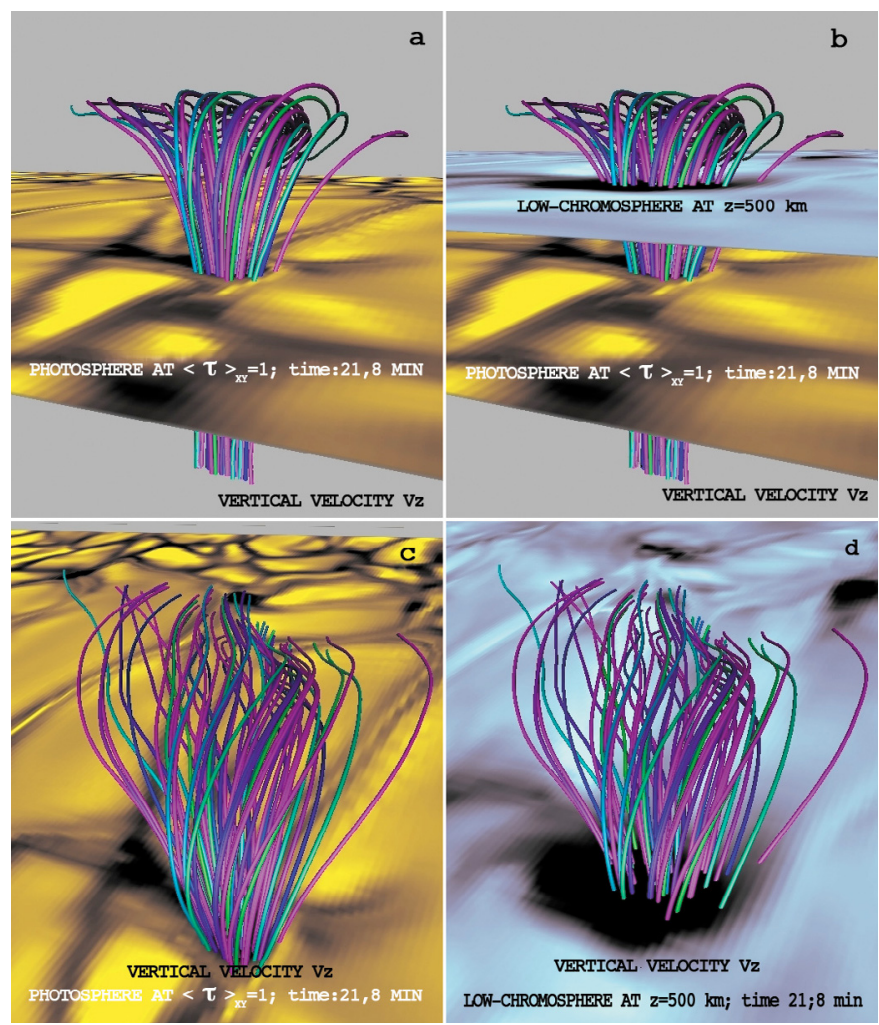

Fig. 15. Representative field lines near a vertical flux concentration $(t=$ 21.8 min, S1 run) with maps for the $v_{z}$, seen from different perspectives. The brown-yellow map is drawn at $\tau=1$ with color scale from -5 (dark brown) to 2 (yellow) $\mathrm{km} \mathrm{s}^{-1}$. The blue-black color map is drawn at $z=500 \mathrm{~km}$ and goes from -10 (black) to 2 (blue) $\mathrm{km} \mathrm{s}^{-1}$.

however, an increasing number of high-temperature and highentropy magnetized elements can be found at each chromospheric height. Studying the entropy changes is particularly interesting, since they reflect the heating of the plasma directly. In the following we consider the entropy changes in the S1 run; however, the basic results in this section are equally valid for the $\mathrm{S} 2$ run.

Figure 16 shows the joint PDF of entropy and temperature for the non-magnetized (upper row) and magnetized (lower row) populations at $t=15.3 \mathrm{~min}$ (left column) and $t=19.4 \mathrm{~min}$ (right column), all of them at a height of $z=800 \mathrm{~km}$. The JPDF for the non-magnetized component reflects the thermal structure of the initial chromosphere with its cool and hot components, as discussed in Sect. 4.1. The vast majority of the elements belong to the cool component (see the dashed red frame at the center), with temperatures around $3750 \mathrm{~K}$ and entropies around $1.95 \times 10^{9} \mathrm{erg} \mathrm{K}^{-1} \mathrm{~g}^{-1}$. The hot component, indicated by the dotdashed red frame at the top, has temperatures above $\sim 6000 \mathrm{~K}$ and comparatively high entropies; it corresponds to a small fraction $(\sim 3 \%)$ of the total number of pixels at that height and time, reflecting that the heating is very localized in space. The low- $T$, low- $s$ material marked with a solid-line square at the bottom of the distribution is not present in the initial chromosphere prior to flux emergence: this is non-magnetized plasma from the lower layers that has been carried along with the emerging tube. On the other hand, at this time the magnetized material occupies $\sim 16 \%$ of the total area of the plane at $z=800 \mathrm{~km}$. The associated JPDF (lower-left panel of Fig. 16) shows that most of the magnetized plasma has very low and uniform temperatures of around $\sim 2700 \mathrm{~K}$ and low entropies typical of the photospheric layers above $z \sim 250 \mathrm{~km}$, which confirms the isentropic evolution and expansion of the emerging magnetic plasma already mentioned in Sect. 4.2.1. 


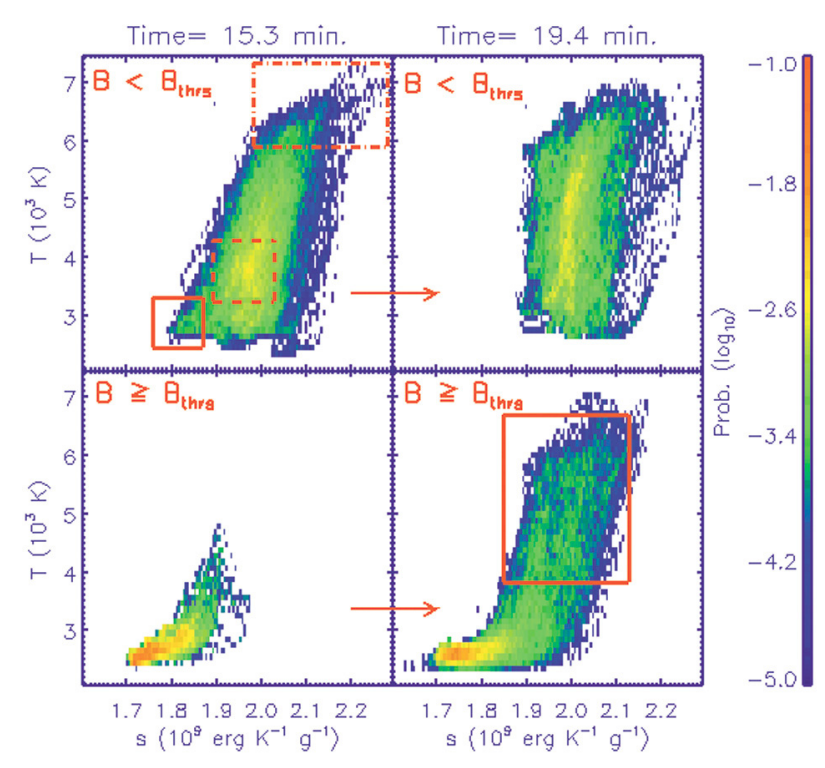

Fig. 16. JPDF for the temperature versus the specific entropy in the midchromosphere $(z=800 \mathrm{~km})$, at two characteristic times in the $S 1$ experiment. Separate panels are given for weakly (top) and strongly (bottom) magnetized pixels, with the mutual boundary set at $B_{\text {thrs }}=10 \mathrm{G}$. The color code corresponds to the logarithm of the probability density.

The most remarkable aspect of the subsequent thermodynamic evolution of the magnetized plasma in the chromosphere is the increase in the temperature and entropy in localized regions that suggests that an effective heating mechanism is taking place. This is reflected in the JPDF of Fig. 16 through the appearance at $t=19.4$ min (lower-right panel) of a high- $T$, high- $s$ tail (see solid-line frame). The heating is localized: only $\sim 2.2 \%$ $(\sim 18.9 \%)$ of the magnetized plasma elements at $z=800 \mathrm{~km}$ have temperatures above $6000 \mathrm{~K}(4000 \mathrm{~K})$. By comparing the JPDF for magnetized and unmagnetized elements (two rightmost panels), it also looks as if the hot tail of the former is progressively adopting a shape similar to the latter. The heating is also noticeable in terms of the averages: compared to the previous time, the mean temperature and entropy are shifted upward by $\Delta T \sim 500 \mathrm{~K}$ and $\Delta s \sim 0.05 \cdot\left\langle s_{\text {phot }}\right\rangle_{x y, t=0}$, respectively.

\subsection{High-temperature points}

A prominent chromospheric thermal structure mentioned in Sect. 4.2.2 is a collection of high-temperature spots. They coincide with concentrations of strong supersonic downflows and vertical fields that extend from the low-chromosphere to the photosphere. These high- $T$ points are associated with the highest temperatures and entropies found in the magnetic plasma and are the main evidence of chromospheric heating reported in the previous section. They appear in both the S1 and S2 runs with similar behavior and global properties.

To quantify their properties, we present in Fig. 17 the JPDF between various physical quantities and the specific entropy for three different chromospheric heights. The chosen sample consists of magnetized pixels with $|B|>10 \mathrm{G}$ and $T>4000 \mathrm{~K}$. The high- $T$ spots coincide with the sparse population of high-entropy pixels (say $s>1.95 \times 10^{9} \mathrm{erg} \mathrm{K}^{-1} \mathrm{~g}^{-1}$ ) in the figure. Looking at the two topmost rows of panels, this population is seen to have almost vertical magnetic field with intensity distributed close to a preferential value (from $300 \mathrm{G}$ at $z=500 \mathrm{~km}$, to $170 \mathrm{G}$ at $z=700 \mathrm{~km}$ ). This agrees with the gradual fading of the vertical

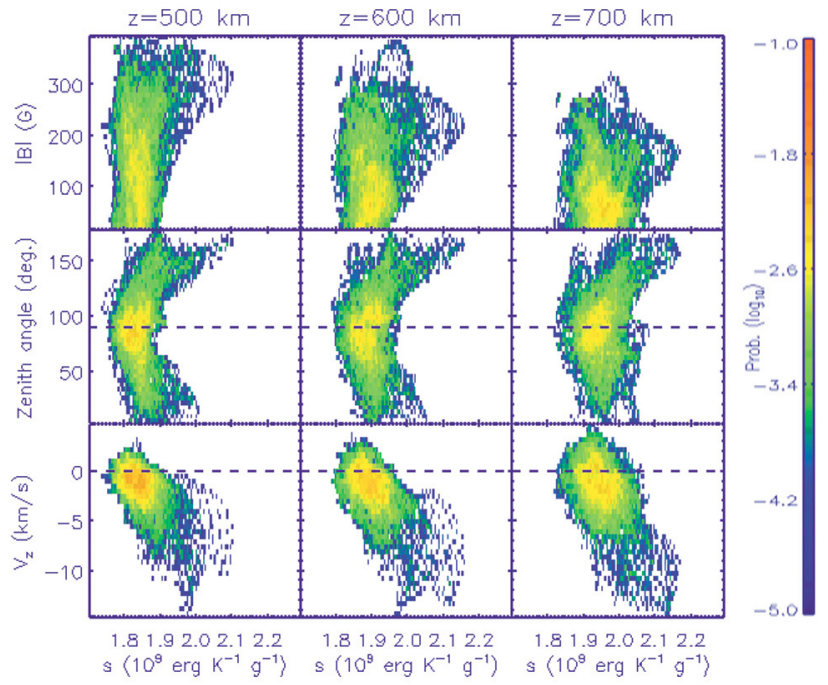

Fig. 17. JPDFs for entropy and magnetic field (intensity and zenith angle, two top rows) and entropy and $v_{z}$ (bottom row) at three heights in the low chromosphere $(t=21.2 \mathrm{~min}, \mathrm{~S} 1 \mathrm{run})$. The color code corresponds to the logarithm of the probability density. To study the chromospheric heating, the sample has been limited to pixels with $|B| \geq B_{\mathrm{thrs}}=10 \mathrm{G}$ and $T>4000 \mathrm{~K}$.

field concentrations toward larger heights in the chromosphere reported in Sect. 4.3. The JPDF of the vertical velocity (bottom row of Fig. 17) shows that the high-entropy tail of the distribution corresponds to strong downflows, which, especially at the higher levels, have supersonic values $\left(\left|v_{z}\right|>10 \mathrm{~km} \mathrm{~s}^{-1}\right)$. The horizontal velocity for these pixels is between 3 and $4 \mathrm{~km} \mathrm{~s}^{-1}$.

In our calculations, we have identified two types of hightemperature points, which we shall refer to as type $A$ and type $B$ in the following. They have similar properties in the chromosphere, but differ strongly in their appearance in the photosphere. We have also found Hinode observational data that correspond to either type of point. All this is illustrated in Fig. 18. The points of type A (block of 4 panels on the left) appear in the experiment (leftmost column) as concentrated temperature enhancements both in the low chromosphere ( $z=500 \mathrm{~km}$; top row) and in the photosphere ( $\tau=1$; lower row). In the low chromosphere, the temperature reaches $\sim 6200 \mathrm{~K}$; the high- $T$ point is cospatial with a concentrated region of high-speed downflow $\left(\left|v_{z}\right|>8 \mathrm{~km} \mathrm{~s}^{-1}\right)$ and vertical fields. The points of type B (block of 4 panels on the right) have similar properties at that height; minor differences are: the entropy, temperature, and downflow speeds are somewhat higher for the latter type (e.g., the downflows are in excess of $10 \mathrm{~km} \mathrm{~s}^{-1}$ for B-type points). In contrast, the differences between the two types are remarkable in the photosphere, and this serves as a basis for the classification. At the visible surface, point A is still well defined and located roughly at the same horizontal position as in the chromosphere (leftmost panel in the bottom row); it is associated with a prominent $\mathrm{kG}$ concentration of vertical fields and strong downflows with velocities of more than $5 \mathrm{~km} \mathrm{~s}^{-1}$. The photospheric temperature panel for point B (bottom-left panel in the right-hand block), instead, shows no bright point feature; magnetograms and Dopplergrams at that height and position only show a disrupted concentration of vertical fields and downflows. The right column in each block shows Hinode images that seem to match those two types of points. They correspond to simultaneous Ca-II H (top) and $G$-band (bottom) observations taken on November 2, 2006 at disk center (a movie is available for these 


\section{Type A}

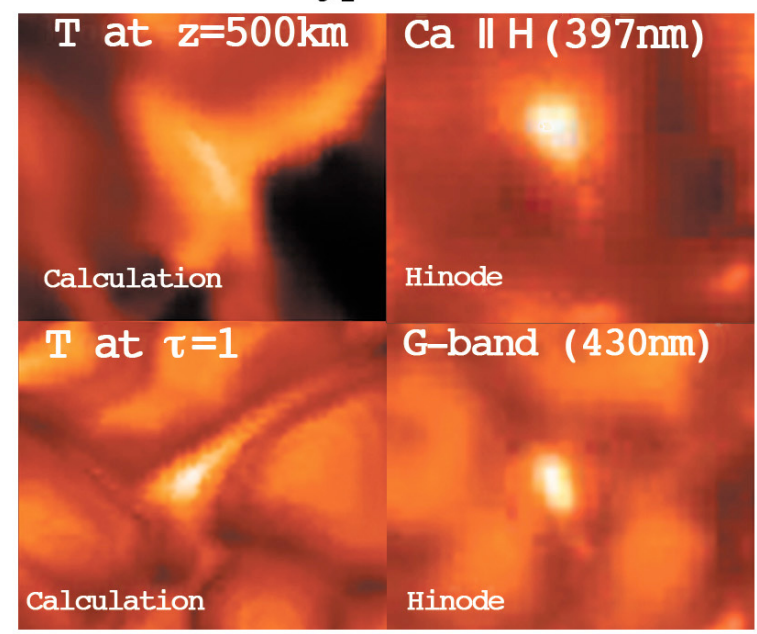

Type B

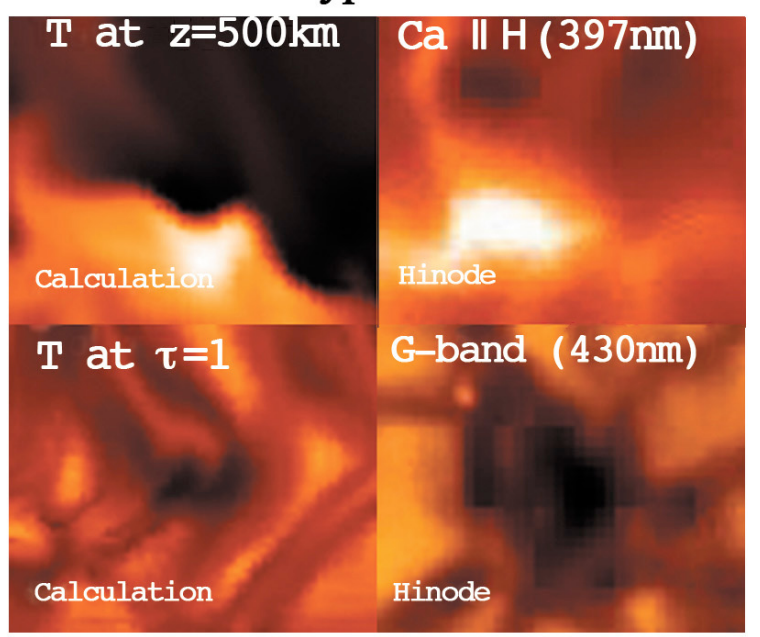

Fig. 18. Two types of high-temperature points: type A (left block) and type B (right block). The left column in each block are temperature maps from the experiments at $z=500 \mathrm{~km}$ (top) and $\tau=1$ (bottom). The right column are observations from Hinode, one (left block) that shows a clear point-like structure in Ca-II H (top panel) and $G$-band (photosphere) and a second one (right block) which lacks a clear photospheric counterpart.

observations $^{1}$ ), showing, therefore, the situation at low chromospheric and photospheric heights, respectively. The match between chromospheric and photospheric brightenings in the observations on the left is apparent. In the right-hand block, there is instead no bright feature in $G$-band that would correspond to the Ca-II H image. The high-temperature points are especially prominent in the low chromosphere. They are also seen in the mid-chromosphere (above $z=750 \mathrm{~km}$ ) but with a more extended and disrupted morphology and not as a point-like structure.

Further observational support can be derived from the results of Shimizu et al. (2008), which are in qualitative and rough quantitative agreement with the features of the A-type points just described. They found high-speed downflows at small concentrations of vertical magnetic field in the quiet Sun photosphere and chromosphere. The observations show point-like transient brightenings in $\mathrm{Ca}-\mathrm{II} \mathrm{H}$ at the position of observed supersonic downflows in the chromosphere, coinciding with a bright point observed in $G$-band. They suggest that the chromospheric bright point may be a signature of a local transient heating. A comparison between our numerical results and the observations is tentative only without detailed spectral synthesis calculations from our experiments. Still, the remarkable similarities between the description in this paper and the transient $\mathrm{Ca}$-II $\mathrm{H}$ brightenings of Shimizu et al. (2008) seem to indicate that both may correspond to the same type of feature in the Sun.

There is evidence in our experiments that points to the shocks as the main mechanism of chromospheric heating in the high-temperature points (see Sect. 5.3). The shocks probably form in the photosphere at the locations of the strong vertical field concentrations as a result of a rebound of the material flowing down from the low chromosphere (Grossmann-Doerth et al. 1998). Following those authors, if the shock is very strong it leads to a suppression of the field intensification that had formed by means of convective collapse. This could explain the high$T$ points of type B, which do not show an intensification at the photosphere but, in contrast, have the highest heating rates, temperature, and downflow velocities in the low chromosphere. If the shock is less strong, it does not lead to the destruction of the

${ }^{1}$ http://solarb.msfc.nasa.gov/movies/cagb_20061102.mpg field concentration. This could explain the type-A points, which have moderate heating in the chromosphere correlated with a strong bright point at the visible surface. Strong upflows and upgoing shock fronts moving in vertical field concentrations in the quiet-Sun photosphere have already been reported in the literature (Bellot Rubio et al. 2001; Socas-Navarro \& Manso Sainz 2005). The results shown in the present paper suggest that they must occur in small-scale emergence episodes and can play an important role as a heating mechanism of the magnetized plasma in the low chromosphere.

\section{Lagrangian study}

An effective way to gain insight into the distinctive behavior of the rising magnetized plasma elements is to use Lagrange tracers, i.e., to pursue individual plasma elements in time as they move in the interior and atmosphere. We calculated the trajectory of a total of 9000 Lagrange particles, which, at the time of arrival of the magnetic flux at the surface $(t \sim 8 \mathrm{~min})$, are uniformly distributed in square patches of $6 \mathrm{Mm} \times 4 \mathrm{Mm}$ $(x, y:[-3,3] \times[-2,2] \mathrm{Mm})$ within planes separated by $50 \mathrm{~km}$ in the range $0-500 \mathrm{~km}$ below the visible surface. The Lagrangian integration was carried out for the $\mathrm{S} 2$ run $(\Omega$-loop).

\subsection{Lagrangian evolution in the photosphere}

In Fig. 19, we compare the profiles of $T$, $s$, and $\rho$ for the trajectories of two particles starting in an upflow region in the convection zone. The blue path corresponds to a magnetic particle initially located at $z=-440 \mathrm{~km}$ with $|B| \sim 1300 \mathrm{G}$. The yellow path corresponds to a non-magnetized particle, initially at $z=-160 \mathrm{~km}$ with a horizontal offset of $\sim 2 \mathrm{Mm}$ from the axis of the tube. The red-dashed sections identify the phases where the radiative term heats the particle $\left(Q_{\mathrm{rad}}>0\right)$. Those particles were chosen because they show similar behavior in their rise to the surface and do not rise beyond the photospheric heights. Their return to the interior differs markedly, however, with the magnetic particle undergoing a process of convective collapse. 


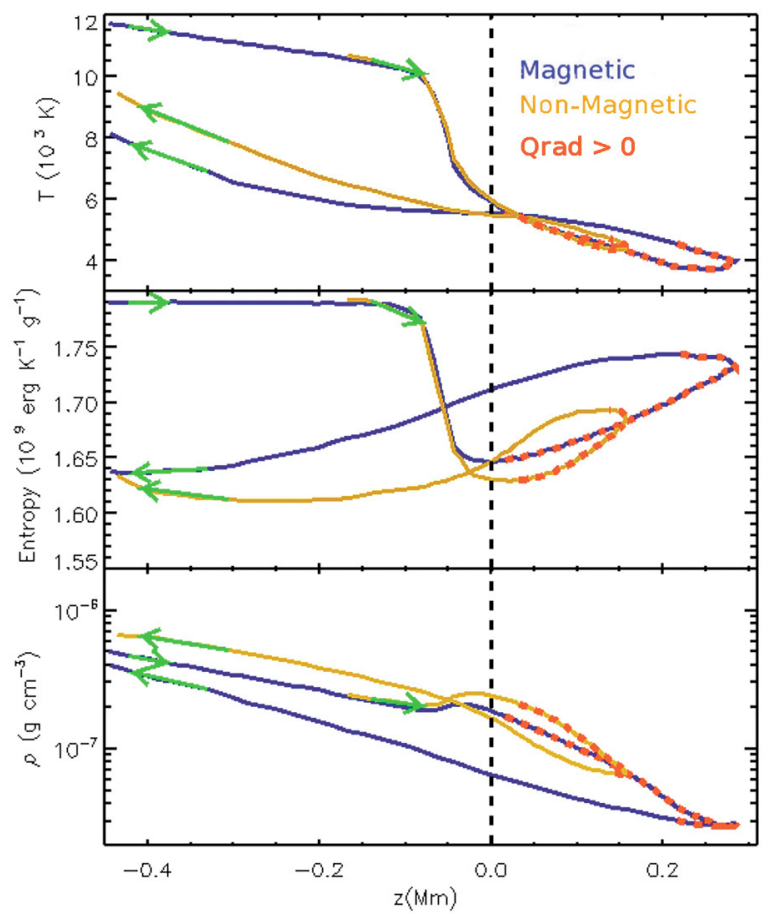

Fig. 19. Comparison of the Lagrangian profiles of temperature (upper panel), specific entropy (mid panel) and density (lower panel) for a magnetic (blue) and non-magnetic (yellow) particle that reach the surface. The green arrows indicate the direction of motion. In the red-dashed sections, the plasma is being heated by the radiation field $\left(Q_{\mathrm{rad}}>0\right)$.
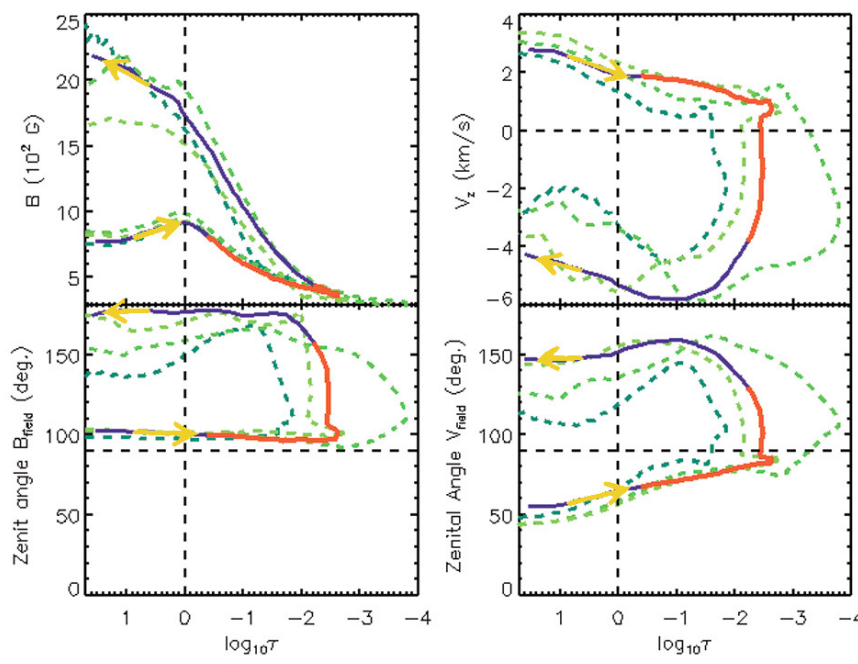

Fig. 20. Lagrangian evolution of $|B|$ (upper-left), $v_{z}$ (upper-right), and of the zenith angle for the $B$ (lower-left) and $v$ (lower-right) vectors for a magnetic particle that undergoes convective collapse. Yellow arrows indicate the direction of motion. Solid-red curve sections mark where the plasma is being radiatively heated. Plasma elements in a $1 \mathrm{Mm}^{2}$ neighborhood of that particle at the surface also suffer convective collapse, as shown by their Lagrange trajectories, drawn as green dashed curves.

The behavior of the non-magnetic tracer (yellow curves) conforms to the description of Cheung et al. (2007b) when explaining the origin of the reverse granulation. Three stages are clearly identified: a) radiative cooling close to the visible surface; b) temperature decrease and radiative heating, while rising and expanding in the atmosphere; and c) overturn and descent into the convection zone. As for the non-magnetic particle, the magnetized tracer rises adiabatically in the convection zone (e.g., Stein \& Nordlund 1998) and experiences strong radiative cooling at the surface, which causes a pronounced decrease in the entropy and temperature of the plasma, and a moderate decrease in the specific volume; this tracer can resist the latter to some extent by means of the magnetic pressure, so that the density does not increase as much as for the non-magnetic particle. Lower density leads to reduced opacity, so that also the cooling rate $Q_{\mathrm{rad}} / \rho$ and the loss of entropy are lower for the magnetic elements. Higher in the atmosphere, both particles experience a large expansion and an associated decrease in temperature, which brings them below the radiative equilibrium value. Radiative heating and entropy growth are evident (as indicated by the red dashes in Fig. 19). To be representative of the average behavior (Sect. 5.2.1), the magnetized particle chosen for this figure reaches a greater height in the atmosphere than the other one. It is then heated for a longer time and its entropy thereby becomes substantially higher. The hysteretic behavior of the temperature or entropy curves that is typical of plasma elements reaching these heights is therefore significantly more marked for the magnetic tracer.

When returning to the surface, the magnetized Lagrange element goes through a process of convective collapse. The convective collapse has been studied using purely theoretical considerations (Parker 1978; Spruit 1979; Schüssler 1990) and numerical simulations (Grossmann-Doerth et al. 1998; Steiner et al. 1998; Takeuchi 1999; Vögler et al. 2005; Cheung et al. 2008). Observationally, Bellot Rubio et al. (2001) and SocasNavarro \& Manso Sainz (2005) found indirect evidence of this process. Direct observational signatures of convective collapse were found by Nagata et al. (2008) from Hinode data. Cheung et al. (2008) study an instance of convective collapse in their flux emergence simulation, considering, in particular, the field strength and brightness of the resulting element compared with the surroundings as one would observe it and the small Wilson depression that develops within the collapsed magnetic element.

In the following, we focus on the Lagrange evolution of plasma elements that suffer a convective collapse, rather than studying the Eulerian aspects of the resulting structure, which can be found in the literature. The process is illustrated in Fig. 20 for the magnetized tracer of Fig. 19. At first, near the visible surface $\left(\log _{10} \tau=0\right)$, the density increase caused by the radiative cooling gives rise to a small intensification of the field strength (Cheung et al. 2007a, 2008). This is followed by a marked field weakening as the particle rises and expands. The trajectory in the atmosphere has a fountain-like appearance, the zenith angles of the velocity vector changing from about $60^{\circ}$ at the surface to horizontal $\left(90^{\circ}\right)$ at the top, and about $150^{\circ}$ when returning to the surface. The magnetic field vector, in turn, is almost horizontal all along the rise (see bottom-left panel of Fig. 20). However, it abruptly changes its orientation and becomes vertical when approaching the downflow section of the trajectory. The plasma element thus loses the support of the magnetic pressure and reaches high downflow speeds of between 4 and $6 \mathrm{~km} \mathrm{~s}^{-1}$. This results in an evacuation of the vertically magnetized plasma, lateral compression, and magnetic field intensification: $|B|$ goes from $400 \mathrm{G}$, at $\log _{10} \tau=-2.2$, to $1750 \mathrm{G}$ at $\log _{10} \tau=0$, (upper-left panel of Fig. 20). The evacuation is particularly noticeable when comparing the density values of magnetized and non-magnetized elements (lower panel of Fig. 19). As a result, the opacity is lower in the magnetic elements and the $\tau=1$ surface is shifted towards the interior. For the Lagrange particles 


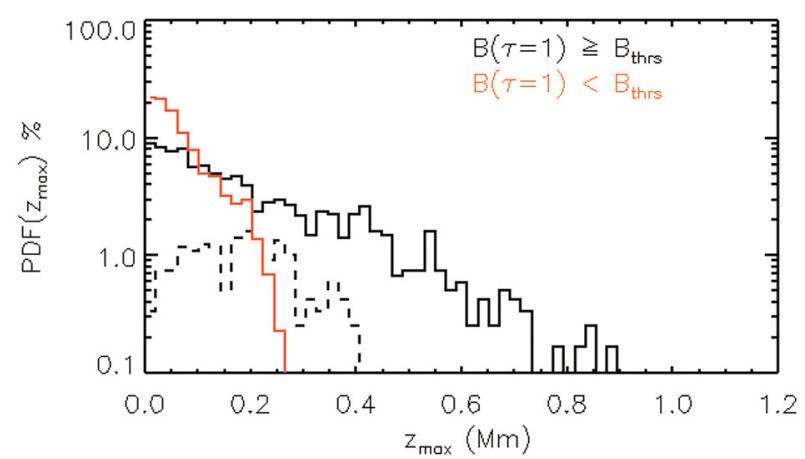

Fig. 21. PDF of the maximum heights $\left(z_{\max }\right)$ reached by different types of particles emerging above the visible surface. Black: magnetized tracers. Red: weakly or non-magnetized tracers. Dashed: separate PDF for tracers that undergo convective collapse.

of Figs. 19 and 20, the downward shift is approximately equal to $175 \mathrm{~km}$. Similar results are found for the plasma elements in a $1 \mathrm{Mm}^{2}$ neighborhood, as is apparent from the green dashed curves in Fig. 20. These results are in good quantitative and qualitative agreement with those of Nagata et al. (2008), which constitute the first direct and clear observational evidence of the formation of strong $\mathrm{kG}$ magnetic flux concentrations produced by the convective instability.

\subsection{Chromospheric layers: Lagrangian statistics}

\subsubsection{Height of emergence of fluid tracers in the atmosphere}

Magnetic flux emergence episodes in the Sun are known to bring magnetized plasma all the way into the corona. On the other hand, under quiet-Sun conditions most of the unmagnetized plasma elements are not expected to rise above the inversegranulation level, a few $100 \mathrm{~km}$ over the visible surface. It is therefore of interest to study the maximum height $\left(z_{\max }\right)$ reached by the Lagrange elements used in this chapter. For those among the 9000 tracers that emerge above $z=0$, we determine the magnetic field strength when they cross the $\tau=1$ level in their upward travel and call it $\left|B\left(\tau^{\text {up }}=1\right)\right|$. We also define a threshold field strength at the surface, $B_{\text {thrs }}=50 \mathrm{G}$. The Lagrange elements are said to be magnetized if $\left|B\left(\tau^{\mathrm{up}}=1\right)\right| \geq B_{\mathrm{thrs}}$ and non-magnetized otherwise. We calculated the separate PDFs of the maximum height $z_{\max }$ reached by either type of element and plotted them in Fig. 21 (black: magnetized elements; red: nonmagnetized). Each PDF is normalized to $100 \%$ of the respective sample. The number of magnetized particles amounts to $13.3 \%$ of the total 9000 Lagrange tracers. Of these, only an $8 \%$ rise above the $500 \mathrm{~km}$ level, and $1.3 \%$ reach heights above $750 \mathrm{~km}$. In contrast, the non-magnetic tracers (red histogram) virtually do not rise beyond the $\sim 200 \mathrm{~km}$ level, i.e., they return to the surface as part of the reverse granulation.

The average maximum height of the distribution for the magnetized particles is $200 \mathrm{~km}$. For many of the particles in the bins around that value, the return to the interior layers takes the form of a convective collapse, as described in Sect. 5.1. To identify those tracers, we selected magnetic particles for which the difference in field strength between the upward and downward crossing of the $\tau=1$ level is larger than the equipartition field in the photosphere $\left(B_{\mathrm{eq}} \sim 500 \mathrm{G}\right)$. We constructed a separate PDF of $z_{\max }$ for those particles (dashed histogram in Fig. 21), normalized to the total number of particles in the magnetic sample. Their
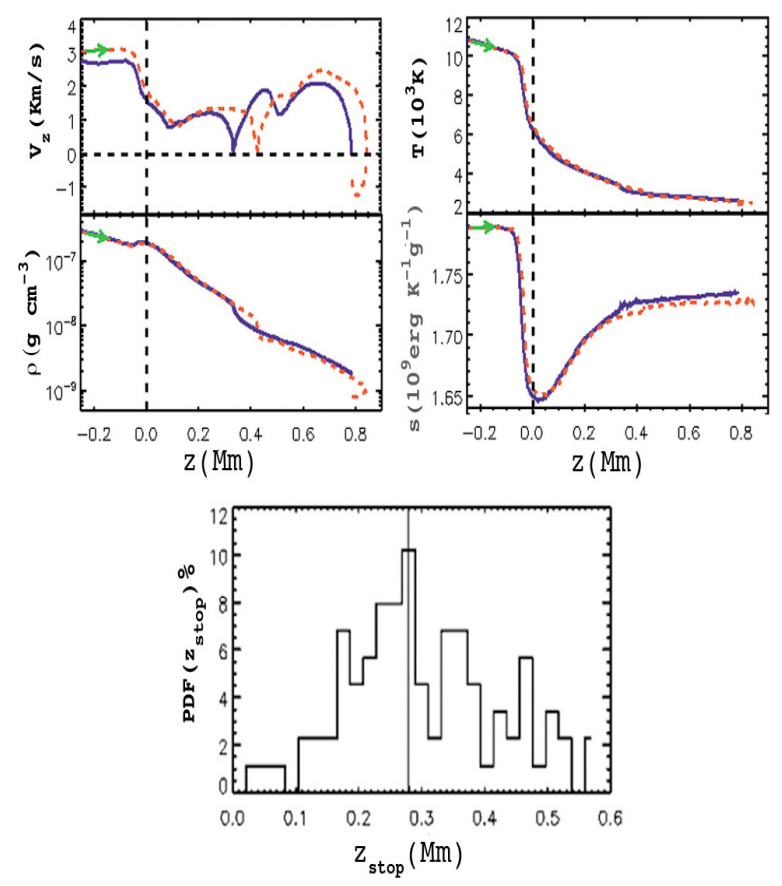

Fig. 22. Temporary stops of magnetized elements rising into the chromosphere. Top panels: Lagrangian profiles of $v_{z}, T, s$ and $\rho$ for two representative particles. Bottom: histogram of the heights of the temporary stops for the particles overshooting the $z=500 \mathrm{~km}$ level. The vertical line marks the median value of the distribution.

distribution is centered on $\sim 180 \mathrm{~km}$. During the experiment, only the tracers with $z_{\max }<400 \mathrm{~km}$ have sufficient time to return to the convection zone and experience a process of convective collapse. This explains the cutoff in the PDF at that height.

The rise of the plasma elements that reach chromospheric levels often occurs in a non-uniform way, with sudden episodes of vertical deceleration that occur at different heights. This is shown in Fig. 22, which contains Lagrangian profiles of the vertical velocity $v_{z}$, temperature $T$, specific entropy $s$, and density $\rho$, for two magnetized particles representative of those that reach mid-chromospheric heights (4 topmost panels). The trajectory of either particle exhibits deceleration episodes at different heights. During the temporary stops, a marked horizontal expansion of the plasma is observed, causing a decrease of the pressure (gaseous and magnetic), temperature and density of the tracers. The plasma $\beta$ also decreases to values close to unity, and the magnetic field strength shows a steep negative gradient. In the discussion (Sect. 6.5), we argue that the magnetic field gradient plays an important role in the upward acceleration of the particles by allowing them to resume the rise, the whole process being strongly indicative of the development of a buoyancy instability (Moreno-Insertis 2006). Figure 22 shows that the subsequent evolution of both tracers occurs under almost isentropic conditions and with a low temperature below $3000 \mathrm{~K}$. For the collection of particles that reach the chromosphere, the statistics of the bottom panel indicates that the setback episodes can occur at different heights mostly above $\sim 200 \mathrm{~km}$.

\subsubsection{Statistical properties at the visible surface of the fluid elements that reach the chromosphere}

Given the small number of Lagrange particles that reach high levels in the atmosphere, it is of interest to ascertain whether those particles are identifiable already at the time when they 

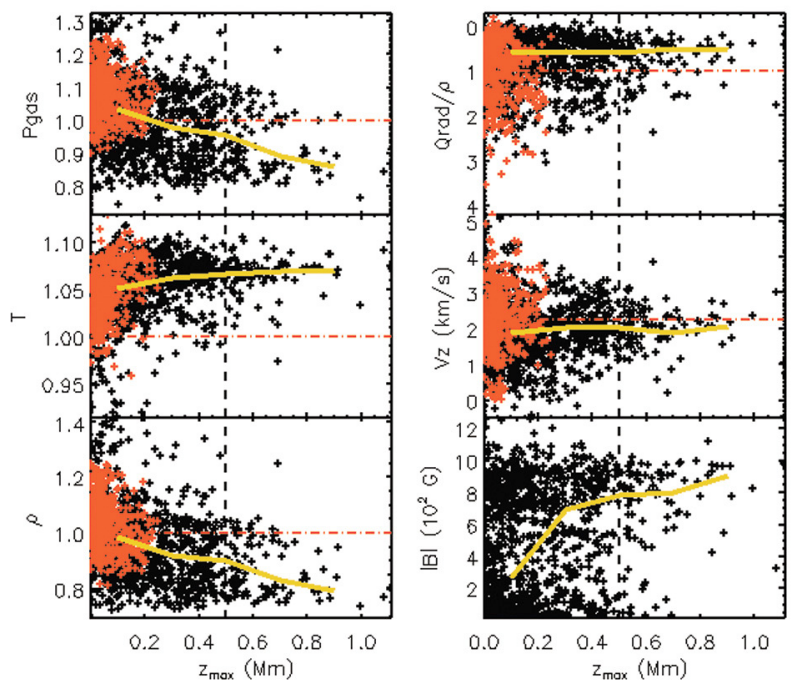

Fig. 23. Relation between the physical properties of rising Lagrangian tracers when crossing the visible surface and the maximum height they reach in their flight. Black points: magnetized particles; red points: non-magnetized particles, chosen with the same criterion as in Fig. 21. Quantities in the panels for $P_{\text {gas }}, T, \rho$ and $Q_{\text {rad }} / \rho$ are normalized to the average for the non-magnetic tracers. The yellow curves show the median values at each height for the magnetic tracers.

cross the photosphere. To this end, we try to identify correlations between the value of different physical variables of the tracers when they cross the level $\tau=1$ in their upward travel and the maximum height they reach in the atmosphere, $z_{\max }$. Figure 23 shows a scatter plot of $z_{\max }$ versus the value of gas pressure, temperature, density, radiative heating, vertical velocity, and magnetic field strength that they have when crossing the $\tau=1$ level. The red and black crosses correspond to field-free and magnetized particles, respectively.

In the photosphere, the radiative transfer is very efficient in regulating the deviations of temperature from the equilibrium: the scatter in the temperature plot amounts to only about 5\% of the mean, whereas the fluctuations in pressure and density reach about $25 \%$ of the average. Only Lagrange elements with $\rho(\tau=1) /\langle\rho\rangle_{\text {non-magnetic }}<1$ reach chromospheric heights; in fact, the density deficit at $\tau=1$ has a clear positive correlation with the maximum height (yellow dot-dashed line) reached by the element. Most tracers that reach the chromosphere also tend to have a high magnetic field strength at $\tau=1$, and there is a global positive correlation between $\left|B\left(\tau^{\mathrm{up}}=1\right)\right|$ and $z_{\max }$. As a consequence of the reduced $\rho$ and $p_{\text {gas }}$, the magnetized particles have lower opacities, and hence lower cooling rates $Q_{\mathrm{rad}} / \rho$ (see uppermost right panel, noting the inverted ordinate scale).

The comparatively low density and high field strength at $\tau=1$ for those Lagrange particles that reach chromospheric heights with, however, similar temperatures, would seem to reveal a standard case of magnetic buoyancy as in the definition of Parker (1955). The correlations mentioned just above would then indicate that the more buoyant elements reach higher levels in the atmosphere. However, one should avoid oversimplifications: the Lagrange elements considered here are not isolated magnetic tubes embedded in field-free plasma. On the other hand, there is a large number of particles with high field strength that do not reach high levels in the atmosphere (Fig. 23, bottom-right). This suggests that other factors, such as the topology of the field and the interaction with the photospheric flows, also play a role in determining the maximum height reached by the plasma elements.

\subsection{Chromospheric layers: shocks in the magnetized volume}

In Sect. 4.5 we identified the shocks as the main mechanism causing the heating at high-temperature points. Figures 24-26 illustrate the formation of a high- $T$ point produced by the passage of a shock using the Lagrangian trajectory of a fluid particle. Figure 24 shows the projection of the actual Lagrangian $\operatorname{track}\left[x_{\mathrm{L}}(t), z_{\mathrm{L}}(t)\right], t<t_{i}$, on color maps of entropy, temperature, and $p_{\text {gas }}$ drawn on the vertical plane $y=y_{\mathrm{L}}\left(t_{i}\right)$ reached by the particle at time $t_{i}$, for different instants $t_{i}$. Figure 25 contains diagrams similar to those of Figs. 19 and 22 showing the value of $\rho, p_{\text {gas }}, p_{\text {total }}, v_{z}, T$, and $B$ at the successive heights reached by the particle. In both Figs. 24 and 25, the red and blue asterisks identify points along the trajectory where $Q_{\text {rad }}>0$ and $Q_{\text {rad }} \leq 0$, respectively.

At $t=19.1 \mathrm{~min}$, the tracer is reached by a shock front in the chromosphere at $z \sim 890 \mathrm{~km}$ above the visible surface (see third column of Fig. 24) causing modifications on its state and trajectory. The Lagrangian evolution of the temperature in Fig. 25 shows a steep growth from $\sim 2360 \mathrm{~K}$ to $\sim 6990 \mathrm{~K}$, accompanied by an increase in entropy (lower row of Fig. 24). The temperature, in fact, increases above the radiative equilibrium value, so that the radiative source term now cools the particle (change from red to blue asterisks). The profiles of gas pressure and density also display a discontinuous increase by a factor of $\sim 6$ and $\sim 2$, respectively. The curves of total pressure and magnetic field strength in Fig. 25, nevertheless, do not show any marked discontinuity at the passage of the shock. The plasma $\beta$, in turn, goes from 0.2 to 2 . The discontinuity, therefore, seems to be a shock, possibly an acoustic shock that propagates along the field lines.

The changes in the trajectory of the particle produced by the shock can be seen in Fig. 24. Before the shock, the particle had just passed the peak of its trajectory with $v_{z} \sim 1 \mathrm{~km} \mathrm{~s}^{-1}$ and $v_{\text {hor }} \sim 10 \mathrm{~km} \mathrm{~s}^{-1}$. The shock kicks the particle upward again, increasing its speed by a factor 4 , before it falls again $(t=19.8 \mathrm{~min})$. The signature of the shock can be clearly seen in the color maps of the figure: the shock front appears as an elongated bright structure propagating diagonally with a supersonic velocity of $\sim 15 \mathrm{~km} \mathrm{~s}^{-1}$. Finally, Fig. 26 shows how the shock front of Fig. 24 creates a high- $T$ point in the chromosphere. The figure contains temperature maps for horizontal planes at the position of the tracer in Fig. 24. The panel at $t=19.0$ min shows a point-like temperature enhancement appearing near the boundary of a cool patch, close to the position of the particle. In the following two panels a high-temperature point is created at the location of the particle after the passage of the shock. Thereafter, the point tends to fade gradually.

\section{Discussion}

We have carried out experiments of the emergence of magnetic flux across granular cells and further up into the chromosphere. The evolution in the photosphere agrees in general terms with the results of Cheung et al. (2007a, 2008), with differences arising because of the choice of parameters for the initial magnetic tubes (especially the field strength and initial depth) in the different experiments. On the other hand, our experiments include some $800 \mathrm{~km}$ above the upper boundary of Cheung et al.'s simulations, which allow us to study the evolution in the pre- and post-emergence atmosphere up to the mid-chromosphere. In the 


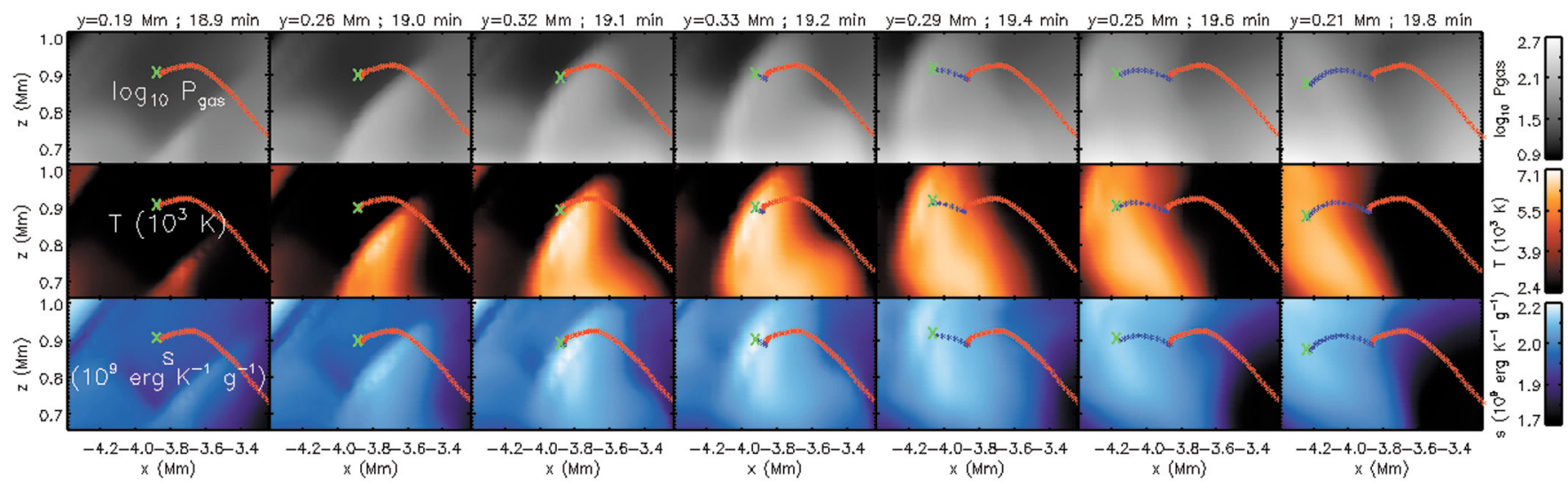

Fig. 24. Magnetized particle hit by a shock front in the chromosphere. The image shows (left to right) a time sequence of the events seen in planes $z x$ at the instantaneous $y$ position of the particle. The color maps represent, from top to bottom, $\log _{10} P_{\text {gas }}, T\left(10^{3} \mathrm{~K}\right)$ and the specific entropy $s$ $\left(10^{9} \mathrm{erg} \mathrm{K}^{-1} \mathrm{~s}^{-1}\right)$. The projection of the previous trajectory of the tracer on the vertical plane is shown in red where $Q_{\text {rad }}>0$ and blue where $Q_{\text {rad }} \leq 0$.
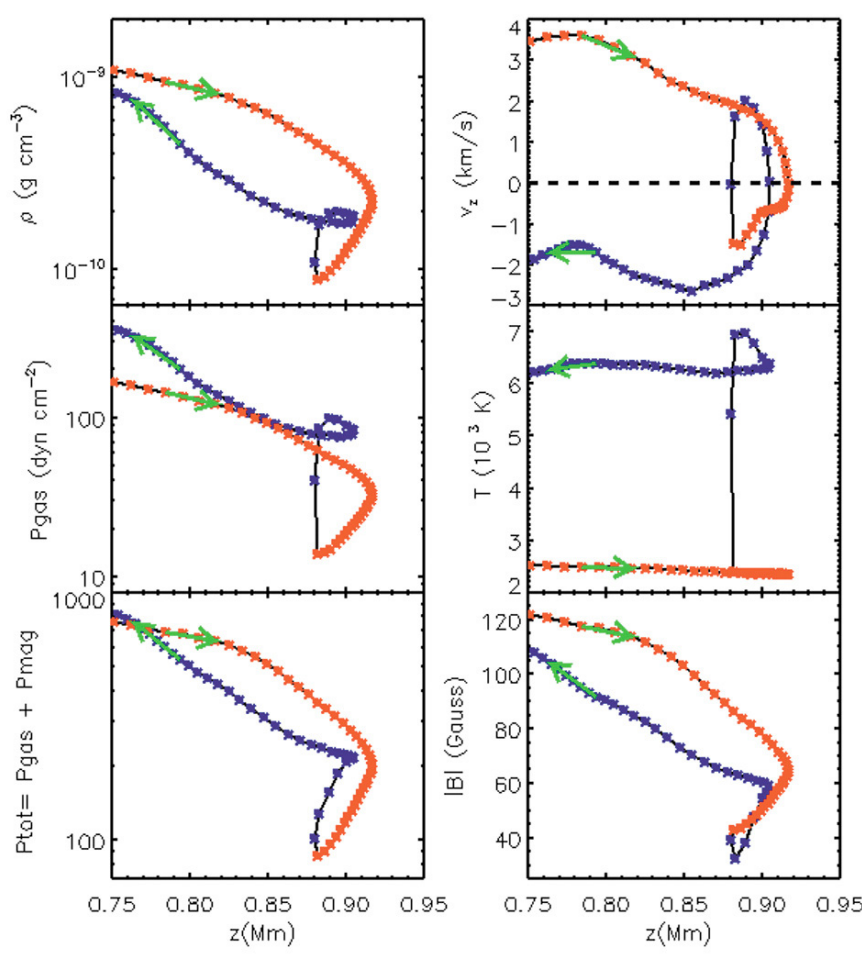

Fig. 25. Lagrangian evolution of a particle hit by a shock. Various physical quantities are shown along the trajectory of the Lagrange particle of Fig. 24 with corresponding blue/red sections in both figures. The green arrows indicate the direction of the motion.

following, we discuss some of the results and assumptions of the present paper.

\subsection{The formation of twisted tubes below granular downflows}

In Sect. 3.4, we reported the formation of twisted horizontal magnetic tubes below new downflow lanes appearing in the middle of a decaying granular cell. The mechanism discovered here seems rather robust and the flux tube thus created can be easily discerned in the experiment. A few remarks can be made about this finding. Retraction of magnetic flux from the lower atmosphere toward the convection zone on a variety of length-scales has been discussed in the literature in connection with flux cancellation events and, more generally, with the general flux budget of the solar surface (see, e.g., the observational results of Chae et al. 2004; Kubo \& Shimizu 2007; Harvey et al. 1999 and the theoretical discussions of Zwaan 1987; Priest 1987; Spruit et al. 1987; Cheung et al. 2008; Ryutova et al. 2003). Different scenarios have been proposed to explain these events. In many cases, the retracting structure is a pre-existing loop of either upward-convex (i.e., $\Omega$-like) or upward-concave (i.e., U-like) shape. These curved loops are assumed to result either directly from flux emergence or by reconnection following the approach of vertically magnetized flux elements of opposite polarity in the atmosphere. In our experiment, the curved shape is formed, instead, out of originally horizontal field because of the appearance of a downflow in the middle of an aging granule. This fits nicely with the recent evidence in favor of a predominance of horizontal fields in the photosphere (e.g., in the internetwork, see Lites et al. 2008; see also the results concerning transient magnetic horizontal fields by Ishikawa \& Tsuneta 2009). In the particular case reported here, the decaying granules have a rather ordered horizontal field given their location in an emerging-flux region. However, the downward-pulling of horizontal fields followed by the subphotospheric reconnection of the resulting vertical legs can yield horizontal twisted tubes in more general cases, with the length of the resulting tube being of the order of the transverse coherence scale of the horizontal fields in the photosphere.

The formation of the tube in a downflow helps it reach deep levels in the granule: as visible in Fig. 6, the tube coincides with an overdense volume and goes down to several hundred $\mathrm{km}$ below the photosphere. A further remark concerns the level of field line twist in the resulting tube. The amount of twist is determined by the reconnection process and depends on the mutual angle between the horizontal field and the orientation of the downflow lane. The Lorentz force in the photosphere is probably not strong enough to influence the direction in which new downflow lanes are created. If so, there should be no preference a priori for any particular angle. Hence, statistically, tubes with a variety of levels of twist should be produced. Finally, the formation of twisted flux tubes below the intergranular lanes just described will not be directly accessible to observations. However, indirect hints can be provided by a) the reconnection upflows detectable (with 


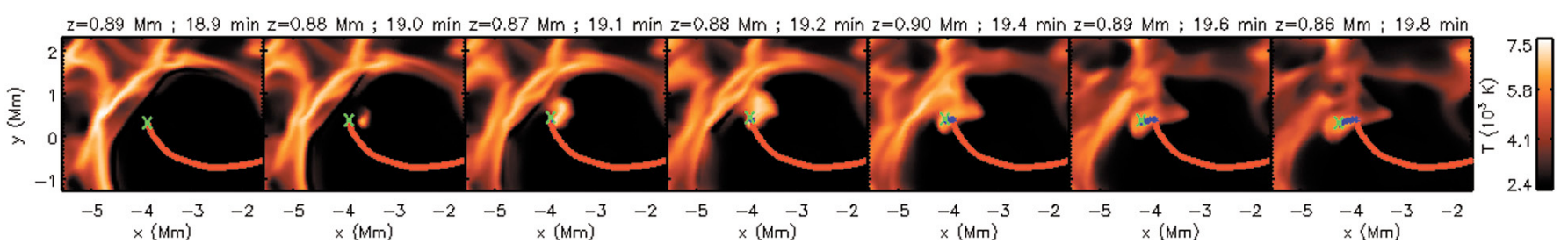

Fig. 26. Formation of a high-temperature point associated with the propagation of a shock in the chromosphere. Shown is the time sequence (left to right) of the temperature map on horizontal planes at the instantaneous height of the particle of Fig. 24. (Symbols for the particle trajectory as in that figure.)

high enough spatial resolution) along the intergranular lanes and b) changes in the topology of the field close to the downflows in the surface layers.

\subsection{The LTE assumption for the radiation transfer in the chromosphere}

For the solution of the radiation transfer equation, we have used the LTE approximation for the source and opacity functions with no resolution in the frequency domain (gray atmosphere). This provides a comparatively simple solution of the radiative problem, and, hence, a higher spatial resolution than with a more complicated approach. The approximation of LTE is acceptable in the photosphere up to a height of several hundred $\mathrm{km}$. The applicability of LTE to the chromosphere is however far more limited. On the plus side, Wedemeyer et al. (2004), using a gray LTE approximation at all heights in their $3 \mathrm{D}$ radiation hydro simulations, find that many basic aspects of the chromospheric dynamics are reproduced approximately in the simulations. On the minus side, this approach neglects two effects that are important in the chromosphere (see Carlsson 2006), namely 1) radiation scattering in strong chromospheric lines and 2) the long timescales for hydrogen ionization/recombination. Concerning the first aspect, Carlsson (2006) points out that a gray-atmosphere approach may reduce the unrealistically high coupling between plasma and radiation field that follows when strong chromospheric lines are included in the opacities while maintaining the LTE assumption and neglecting scattering. The gray LTE approximation may therefore constitute a compromise in this respect. The second problem, however, is more acute. The ionization/recombination time for hydrogen in those heights can be longer than the prevailing dynamical timescales (Kneer 1980; Carlsson \& Stein 1992, 1995, 2002; Leenaarts et al. 2007). Given the complication of its inclusion in 3D MHD codes, a full nonLTE treatment of the hydrogen ionization has not yet been incorporated into 3D radiation-hydrodynamics experiments (see, however, first attempts by Leenaarts \& Wedemeyer-Böhm 2006). The assumption of $\mathrm{H} / \mathrm{H}^{+}$population level equilibrium may lead to too high fluctuations in the level of hydrogen ionization and hence too small temperature fluctuations, e.g., at shock fronts (Carlsson 2006). This should be taken into account when using the quantitative results concerning shock waves in Figs. 24-26.

\subsection{Arrival of flux at the chromosphere: precursor waves}

In general, we expect the chromosphere to react sensitively to any upgoing perturbations originating in the convection zone. In our experiment, we see that the higher levels of the domain (in particular the chromosphere) are perturbed by the emergence of the magnetic tube while the latter is still well within the convection layer. This perturbation takes the form of a running precursor wave that reaches the atmosphere around $t=3.4 \mathrm{~min}$ (i.e., well before the magnetic elements reach the surface). The wave steepens and shocks in the chromosphere, causing a perturbation of the standard local shock pattern. For the S1 experiment, at $z=600 \mathrm{~km}$ the perturbation propagates from a position around $y=0$ (i.e., matching the horizontal location of tube axis) toward the boundaries in the transverse direction, with a pattern reminiscent of the shape of the tube. The S2 run also shows a precursor wave, although the evolution of the associated shocks in the chromosphere is more complex because of the geometry in this case. The particular shape of this precursor wave clearly depends on the initial conditions of our experiment. However, if magnetic flux should arrive in localized form at the lower level of the convection cells, it is likely that some sort of precursor signal should become apparent in the chromosphere (in the simulations, and possibly also in observations) minutes before the first magnetic elements reach the visible surface. On the other hand, Martínez-Sykora et al. (2008) noted how the anomalous expansion of the granules in the photosphere in their experiment started some $7 \mathrm{~min}$ before the magnetic tube appears at the surface; the perturbation is also visible a little later at the level of the reversed granulation. We do not see any clear expansion of the granules so long in advance of the arrival of the magnetic flux (cf. Sect. 3.2), but this may be due to the differences in the initial parameters of the experiments (field strength, field distribution).

\subsection{Hot structures in the chromosphere: adiabatic versus non-adiabatic temperature increase}

In Sect. 4, we have shown how the arrival of magnetized plasma at chromospheric heights causes a modification of the pre-existing structure with the appearance of cool magnetized patches, hot filaments in their periphery, and a number of hightemperature points appearing at the edges of the cool patches. Furthermore, we have shown (Sect. 4.4) that the increase in temperature of the chromospheric structures is due to non-adiabatic (i.e., true) heating (see Fig. 16), since it is associated with an increase in the entropy of the plasma elements. It may be interesting to compare these results with the findings of MartínezSykora et al. (2008). They detected generally higher temperatures at the boundary of the magnetized cells in the lower chromosphere and, also, high- $T$ points, especially after the magnetic tube had gone past the low chromospheric heights. While their high- $T$ points were correlated with large values of magnetic field and vertical velocity (like ours), their high temperature was attributed to the compression of the plasma, the ohmic and viscous heating sources being of lesser importance. This is in contrast to our results, and may be indicative of the different nature of the structures found in our respective experiments.

Concerning the sources of entropy that cause the heating of the hot chromospheric plasma in our experiment, we have documented instances of the creation of the high- $T$ points via shock propagation from lower levels (as in Figs. 24, 26 and 25). 
We have also found that the hot filaments surrounding the cool patches acquire their high temperature by non-adiabatic means, although in this case they seem to have been heated through ohmic dissipation: they are located in places with high magnetic gradients, and therefore, large electrical currents. In either case, we do not find evidence of radiative heating, given the optically thin nature of the chromospheric domain in our simulation. Further research will be needed to ascertain the actual mechanism of heating; on the other hand, the growth of entropy in the plasma seems an inescapable conclusion from our data.

We have also detected the presence of two types of high- $T$ points, which have similar properties in the chromosphere but widely different appearance in the visible surface, namely concentrated and cospatial with the chromospheric feature (type A), or dispersed and inconspicuous (type B). We have also found evidence of these two types of points in Hinode observations. For those of type B, a tentative identification has been made with the magnetic features proposed by Grossmann-Doerth et al. (1998) in which a rebound of the downflowing material may lead to destruction of the photospheric concentration, a phenomenon that also has some observational support.

\subsection{The rise into the atmosphere: temporary stops along the rise}

We have found statistical evidence (Sect. 5.2.1) that the rise of the magnetized plasma in the low atmosphere proceeds in the form of a series of jumps with stops in-between instead of in a continuous fashion. There has been some discussion in the literature about the difficulty of the rise of the emerging magnetized plasma through the photosphere. On the one hand, in many experiments that neglect the coupling of the plasma with the radiation field, the authors report that the rising plasma suffers a temporary stop at the photosphere (see Magara 2001 in 2D; Archontis et al. 2004 in 3D). This can be explained by the suppression of buoyancy instabilities in magnetized plasma associated with the subadiabatic stratification of the photospheric layers (see Moreno-Insertis 2006): using the stability criterion of Newcomb (1961) and Acheson (1979), one can show that the simplest buoyancy instabilities can develop (thus allowing the further rise) if sufficient magnetic flux piles up high enough in the photosphere so that the plasma $\beta$ decreases and the product $|\beta \delta|$ becomes low enough (where $\delta$ is the customary superadiabatic excess in the logarithmic temperature gradient, $\left.\delta=\nabla-\nabla_{\mathrm{ad}}\right)$. On the other hand, still in experiments that disregard the coupling to the radiation field, the axis of the emerging tube does not rise above the photosphere, i.e., it is only the top layers of the tube that reach the higher atmosphere. This has led to discussions about the mechanisms that could allow entire tubes to escape into the corona (see Manchester et al. 2004; Hood et al. 2009).

In our experiment, as in others that include radiative transfer (e.g., Cheung et al. 2007a), the entire magnetic tube crosses the photosphere unimpeded, aided by the extra push provided by the convective upflows. However, we find a novel feature: the rise in the atmosphere proceeds through a series of temporary stops taking place at levels mostly between 200 and $500 \mathrm{~km}$ (Fig. 22). It is interesting that those heights are immediately above the level of the inverse granulation in the non-magnetic convection; hence they correspond to heights where no extra push can be expected from the convection cell. The stops in our experiment are accompanied by a sideways expansion and lowering of the plasma $\beta$. This indicates that the local stops can indeed be produced by the mechanism of flux pile-up, followed by the launching of a buoyancy instability, as discussed by Moreno-Insertis (2006).

\subsection{Concluding remarks}

Some of the results presented in this paper could be further verified and extended by obtaining synthetic spectra, including Stokes parameters, from the data in our computational box. Also, the wealth of recent observational data, especially from the Hinode mission is also amenable to direct comparison with individual aspects of the evolution obtained in this experiment. Both aspects go beyond the scope of the present publication and are to be tackled as part of future work.

Acknowledgements. Financial support by the European Commission through the SOLAIRE Network (MTRN-CT-2006-035484) and by the Spanish Ministry of Research and Innovation through projects AYA2007-66502 and CSD200700050 are gratefully acknowledged, as are the computer resources, technical expertise and assistance provided by the MareNostrum (BSC/CNS, Spain) and LaPalma (IAC/RES, Spain) supercomputer installations. The authors are grateful to A. Vögler for his support and hospitality during a stay of A. T. A. at the Univ. of Utrecht and to him and M. Schüssler for permission to use the MURaM code. We are grateful to the authors of the VAPOR visualization software (NCAR, USA, www.vapor.ucar.edu), for the use of the package and for specific help with its installation. Thanks are also due to the Hinode mission for permission to use observation images. Hinode is a Japanese mission developed and launched by ISAS/JAXA, with NAOJ, NASA and STFC as partners, and co-operated with ESA and NSC. We are grateful to M. C. M. Cheung, V. Martinez Pillet, J. A. Bonet Navarro, J. Trujillo Bueno, E. Khomenkho and R. Hammerschlag for interesting discussions.

\section{References}

Abbett, W. P., \& Fisher, G. H. 2003, ApJ, 582, 475

Acheson, D. J. 1979, Sol. Phys., 62, 23

Arber, T. D., Haynes, M., \& Leake, J. E. 2007, ApJ, 666, 541

Archontis, V., Moreno-Insertis, F., Galsgaard, K., Hood, A. W., \& O'Shea, E. 2004, A\&A, 426, 1047

Archontis, V., Moreno-Insertis, F., Galsgaard, K., \& Hood, A. W. 2005, ApJ, 635,1299

Bellot Rubio, L. R., Rodríguez Hidalgo, I., Collados, M., Khomenko, E., \& Ruiz Cobo, B. 2001, ApJ, 560, 1010

Carlsson, M. 2006, in Solar MHD Theory and Observations: A High Spatial Resolution Perspective, ed. J. Leibacher, R. F. Stein, \& H. Uitenbroek, ASP Conf. Ser., 354, 291

Carlsson, M., \& Stein, R. F. 1992, ApJ, 397, L59

Carlsson, M., \& Stein, R. F. 1995, ApJ, 440, L29

Carlsson, M., \& Stein, R. F. 1997, ApJ, 481, 500

Carlsson, M., \& Stein, R. F. 2002, ApJ, 572, 626

Centeno, R., Socas-Navarro, H., Lites, B., et al. 2007, ApJ, 666, L137

Chae, J., Moon, Y.-J., \& Pevtsov, A. A. 2004, ApJ, 602, L65

Cheung, M. C. M. 2006, Ph.D. Thesis, Univ. Göttingen

Cheung, M. C. M., Schüssler, M., \& Moreno-Insertis, F. 2007a, A\&A, 467, 703

Cheung, M. C. M., Schüssler, M., \& Moreno-Insertis, F. 2007b, A\&A, 461, 1163

Cheung, M. C. M., Schüssler, M., Tarbell, T. D., \& Title, A. M. 2008, ApJ, 687, 1373

De Pontieu, B. 2002, ApJ, 569, 474

Dorch, S. B. F., Gudiksen, B. V., Abbett, W. P., \& Nordlund, A. 2001, A\&A, 380,734

Fan, Y. 2001, ApJ, 554, L111

Fan, Y., Abbett, W. P., \& Fisher, G. H. 2003, ApJ, 582, 1206

Galsgaard, K., Moreno-Insertis, F., Archontis, V., \& Hood, A. W. 2005, ApJ, 618,153

Galsgaard, K., Archontis, V., Moreno-Insertis, F., \& Hood, A. W. 2007, ApJ, 666,516

Grossmann-Doerth, U., Schuessler, M., \& Steiner, O. 1998, A\&A, 337, 928

Hammerschlag, R., Bettonvil, F., \& Sütterlin, P. 2007, Dutch Open Telescope, Observations of 21 Oct 1999

[ftp://dotdb.phys.uu.nl/1999/1999-10-21/AR08737b/]

Harvey, K. L., Jones, H. P., Schrijver, C. J., \& Penn, M. J. 1999, Sol. Phys., 190, 35

Harvey, J. W., Branston, D., Henney, C. J., \& Keller, C. U. 2007, ApJ, 659, L177

Hood, A. W., Archontis, V., Galsgaard, K., \& Moreno-Insertis, F. 2009, A\&A, submitted 
Ishikawa, R., \& Tsuneta, S. 2009, A\&A, 495, 607

Ishikawa, R., Tsuneta, S., Ichimoto, K., et al. 2008, A\&A, 481, L25

Judge, P. 2006, in Solar MHD Theory and Observations: A High Spatial Resolution Perspective, ed. J. Leibacher, R. F. Stein, \& H. Uitenbroek, ASP Conf. Ser., 354, 259

Kneer, F. 1980, A\&A, 87, 229

Kubo, M., \& Shimizu, T. 2007, ApJ, 671, 990

Kubo, M., Shimizu, T., \& Lites, B. W. 2003, ApJ, 595, 465

Kusano, K., Moriyama, K., \& Miyoshi, T. 1998, Phys. Plasmas, 5, 2582

Leenaarts, J., \& Wedemeyer-Böhm, S. 2006, A\&A, 460, 301

Leenaarts, J., Carlsson, M., Hansteen, V., \& Rutten, R. J. 2007, A\&A, 473, 625

Lites, B. W. 2009, Space Sci. Rev., 144, 197

Lites, B. W., Low, B. C., Martinez Pillet, V., et al. 1995, ApJ, 446, 877

Lites, B. W., Leka, K., Skumanich, A., Martinez Pillet, V., \& Shimizu, T. 1996, ApJ, 460, 1019

Lites, B. W., Skumanich, A., \& Martinez Pillet, V. 1998, A\&A, 333, 1053

Lites, B. W., Kubo, M., Socas-Navarro, H., et al. 2008, ApJ, 672, 1237

Magara, T. 2001, ApJ, 549, 608

Magara, T., \& Longcope, D. W. 2001, ApJ, 559, L55

Magara, T., \& Longcope, D. W. 2003, ApJ, 586, 630

Manchester, W. 2001, ApJ, 547, 503

Manchester, W., Gombosi, T., DeZeeuw, D., \& Fan, Y. 2004, ApJ, 610, 588

Martinez Gonzalez, M. J., \& Bellot Rubio, L. 2009, ApJ, accepted

Martinez Pillet, V., Lites, B., Skumanich, A., \& Degenhardt, D. 1994, ApJ, 425, L113

Martínez-Sykora, J., Hansteen, V., \& Carlsson, M. 2008, ApJ, 679, 871

Matsumoto, R., \& Shibata, K. 1992, PASJ, 44, 167

Matsumoto, R., Tajima, T., Shibata, K., \& Kaisig, M. 1993, ApJ, 414, 357

Matsumoto, R., Tajima, T., Chou, W., Okubo, A., \& Shibata, K. 1998, ApJ, 493, L43

Miyagoshi, T., \& Yokoyama, T. 2004, ApJ, 614, 1042

Moreno-Insertis, F. 1983, A\&A, 122, 241

Moreno-Insertis, F. 2006, in Solar MHD Theory and Observations: A High Spatial Resolution Perspective, ed. J. Leibacher, R. F. Stein, \& H. Uitenbroek, ASP Conf. Ser., 354, 183

Moreno-Insertis, F., Galsgaard, K., \& Ugarte-Urra, I. 2008, ApJ, 673, L211

Murray, M. J., Hood, A. W., Moreno-Insertis, F., Galsgaard, K., \& Archontis, V. 2006, A\&A, 460, 909
Murray, M. J., van Driel-Gesztelyi, L., \& Baker, D. 2009, A\&A, 494, 329

Nagata, S., Tsuneta, S., Suematsu, Y., et al. 2008, ApJ, 677, L145

Newcomb, W. 1961, Phys. Fl., 4, 391

Nordlund, A. 1982, A\&A, 107, 1

Nozawa, S., Shibata, K., Matsumoto, R., et al. 1992, ApJS, 78, 267

Okamoto, T. J., Tsuneta, S., Lites, et al. 2008, ApJ, 673, L215

Orozco Suárez, D., Bellot Rubio, L. R., del Toro Iniesta, J. C., \& Tsuneta, S. 2008, A\&A, 481, L33

Otsuji, K., Shibata, K., Kitai, R., et al. 2007, PASJ, 59, S649

Parker, E. N. 1955, ApJ, 121, 491

Parker, E. N. 1978, ApJ, 221, 368

Priest, E. R. 1987, in The Role of Fine-Scale Magnetic Fields on the Structure of the Solar Atmosphere, ed. E.-H. Schröter, M. Vázquez, \& A. A. Wyller, 297

Rogers, F. J. \& Nayfonov, A. 2002, ApJ, 576, 1064

Rogers, F. J., Swenson, F. J., \& Iglesias, C. A. 1996, ApJ, 456, 902

Rutten, R. J. 2007, in The Physics of Chromospheric Plasmas, ed. P. Heinzel, I. Dorotovič, \& R. J. Rutten, ASP Conf. Ser., 368, 27

Ryutova, M., Tarbell, T. D., \& Shine, R. 2003, Sol. Phys., 213, 231

Schüssler, M. 1990, in Solar Photosphere: Structure, Convection, and Magnetic Fields, ed. J. O. Stenflo, IAU Symp., 138, 161

Shibata, K., Tajima, T., Matsumoto, R., et al. 1989, ApJ, 338, 471

Shimizu, T., Lites, B. W., Katsukawa, Y., et al. 2008, ApJ, 680, 1467

Skartlien, R. 2000, ApJ, 536, 465

Socas-Navarro, H., \& Manso Sainz, R. 2005, ApJ, 620, L71

Spruit, H. C. 1974, Ph.D. Thesis, Univ. Utrecht

Spruit, H. C. 1979, Sol. Phys., 61, 363

Spruit, H. C., Title, A. M., \& van Ballegooijen, A. A. 1987, Sol. Phys., 110, 115

Stein, R. F., \& Nordlund, A. 1998, ApJ, 499, 914

Steiner, O., Grossmann-Doerth, U., Knoelker, M., \& Schuessler, M. 1998, ApJ, 495, 468

Takeuchi, A. 1999, ApJ, 518

van Driel-Gesztelyi, L., \& Culhane, J. 2009, Space Sci. Rev., in press

Vernazza, J. E., Avrett, E. H., \& Loeser, R. 1981, ApJS, 45, 635

Vögler, A., Shelyag, S., Schüssler, M., et al. 2005, A\&A, 429, 335

Wedemeyer, S., Freytag, B., Steffen, M., Ludwig, H.-G., \& Holweger, H. 2004, A\&A, 414, 1121

Yokoyama, T., \& Shibata, K. 1995, Nature, 375, 42

Yokoyama, T., \& Shibata, K. 1996, PASJ, 48, 353

Zwaan, C. 1987, ARA\&A, 25, 83 